UMTG - 1

\title{
Biorthogonal Quantum Systems
}

\author{
Thomas Curtrightt ${ }^{\S \dagger}, *$ and Luca Mezincescu ${ }^{\S, *}$ \\ $\S^{\S}$ Department of Physics, University of Miami, Coral Gables, Florida 33124 \\ ${ }^{\dagger}$ School of Natural Sciences, Institute for Advanced Study, Princeton, New Jersey 08540
}

July 22,2018

\begin{abstract}
Models of PT symmetric quantum mechanics provide examples of biorthogonal quantum systems. The latter incorporporate all the structure of PT symmetric models, and allow for generalizations, especially in situations where the PT construction of the dual space fails. The formalism is illustrated by a few exact results for models of the form $H=(p+\nu)^{2}+\sum_{k>0} \mu_{k} \exp (i k x)$. In some non-trivial cases, equivalent hermitian theories are obtained and shown to be very simple: They are just free (chiral) particles. Field theory extensions are briefly considered.
\end{abstract}

*curtright@physics.miami.edu \& mezincescu@physics.miami.edu

\section{Introduction}

There has been some recent theoretical interest in non-hermitian Schrödinger equations, especially in the guise of "PT symmetric theories" [5, [57]. Therefore, we have considered a few simple soluble examples and explored them in some detail. We believe exact solvability permits the underlying structure to be appreciated more completely. To this end we have found Neumann's work 70 to be remarkably prescient. Although in physics there has long been an interest in complex Hamiltonians for phenomenological purposes 41, 84, [40, quite generally mathematical interest in non-self-adjoint spectral problems pre-dates the invention of quantum mechanics 12. See 37 for a brief survey of the early mathematical history.

Some previous papers have touched on the relevance of biorthogonal systems for PT symmetric models [59, 60, 61, 67, 88. Here we wish to stress the importance of such systems, and their generality, in the context of elementary soluble cases. We consider models whose Hamiltonians are of the form [53, 73,42 , 80, 13, 71, 31, 77.

$$
H=(p+\nu)^{2}+\sum_{k>0} \mu_{k} \exp (i k x)
$$

where the imaginary exponents are of the same sign, and where $\nu$ and $\mu_{k}$ have arbitrary values. Although much is known about the mathematical structure of these models, they tend to be overlooked in the physics literature on PT symmetric theories. We seek to remedy this by offering a thorough discussion of their properties, especially in the case of periodic solutions, for which we believe we make several new observations. We analyze at considerable length the cases of one and two exponentials, and then we indicate how the results extend to the general case. We believe it is almost impossible to over-emphasize the merits of going through such exactly solvable models in complete detail - "the simpler the better" if the important structural features are retained. We end with a brief look at field theoretic extensions. 


\section{Biorthogonal systems}

A sequence of elements $\left\{\psi_{j}\right\}$ and linear functionals $\left\{\Lambda_{k}\right\}$ is said to be biorthogonal (or more precisely, biorthonormal) when

$$
\Lambda_{k}\left(\psi_{j}\right)=\delta_{j k}
$$

See the classic texts by Goursat (45] Vol. III $\S 573$ and $\S 594)$, Banach ([3 Chapter 7), Morse and Feshbach (56) $\S 7.5$ and $\S 8.3$ ), and Gohberg and Krein (44] Chapter 6), as well as 33, 4, 68, 19] 80. The notion of biorthogonality is especially useful when dealing with elements that are analytic functions defined on a contour in the complex plane. In this case, the linear functionals are usually realized as integrals along the contour involving a set of "dual functions" $\chi_{k} \equiv\left(\psi_{k}\right)_{\text {dual }}$, so

$$
\Lambda_{k}\left(\psi_{j}\right)=\int \chi_{k}(z) \psi_{j}(z) d z=\delta_{j k}
$$

However, it is important to stress that a priori there need not be a simple point-by-point relation between the functions and their duals (unlike the standard situation in quantum mechanics). In general, $\chi_{k}(z)$ is not obtained just by conjugation, and differs substantially from $\overline{\psi_{j}(z)}$, even when the contour of integration is a real line segment. Moreover, the normalization and phase of $\psi_{j}$ can be changed, arbitrarily, if a compensating change is made in the normalization and phase of $\chi_{j}$. That is to say, if the system $\left\{\psi_{j}, \chi_{k}\right\}$ satisfies (3), then so does the system $\left\{Z_{j} \psi_{j}, \chi_{k} / Z_{k}\right\}$ (no sum on $j, k$ ) for all non-vanishing, finite choices of the $Z$ s.

For any function in the span of $\left\{\psi_{j}\right\}$, we have

$$
\psi(z)=\sum_{n} c_{n} \psi_{n}(z), \quad c_{n}=\int \chi_{n}(z) \psi(z) d z
$$

assuming the requisite convergence of the sum. For such a $\psi(z)$ we may define, at least formally, an associated dual function by

$$
\psi_{\text {dual }}(z) \equiv \sum_{k} c_{k}^{*} \chi_{k}(z)
$$

While the convergence of the sum for $\psi_{\text {dual }}(z)$ is not necessarily guaranteed by the convergence of that for $\psi(z)$, nevertheless, if it is permissible to interchange $\sum_{k}$ and $\int d z$, then we can make use of (4) and define a norm as

$$
\|\psi\|^{2} \equiv \int \psi_{\text {dual }}(z) \psi(z) d z=\sum_{n}\left|c_{n}\right|^{2}>0
$$

If $\|\psi\|^{2}$ is finite, this allows us to define a bona fide probability distribution on $n$ corresponding to the function $\psi$. The probability of $n$ is just $\left|c_{n}\right|^{2} /\|\psi\|^{2}$. However, it is important to stress that the bilinear $\psi_{\text {dual }}(z) \psi(z)$ might not provide a bona fide probability distribution in the variable $z$, since $\psi_{\text {dual }}(z) \psi(z)$ might be complex. Moreover, $\int \psi_{\text {dual }}(z) z \psi(z) d z$ might be complex for one or both of two reasons: $z$ might be on a complex contour, and $\psi_{\text {dual }}(z)$ is not necessarily $\overline{\psi(z)}$, in general. (Perhaps it is possible to think of $\psi_{\text {dual }}(z) \psi(z)$ as a "complex probability" density [34, 85] but we will not pursue that idea here.)

When $n$ labels the eigenvalue of an operator $L$, such that $L \psi_{n}(z)=\lambda_{n} \psi_{n}(z)$, we may also compute averages of functions $f(L)$ using a procedure analogous to that of conventional quantum mechanics, even if $\psi_{\text {dual }}(z) \neq \overline{\psi(z)}$. These statistical averages are defined to be

$$
\langle f\rangle_{\psi}=\frac{1}{\|\psi\|^{2}} \int \psi_{\text {dual }}(z) f(L) \psi(z) d z=\sum_{n}\left|c_{n}\right|^{2} f\left(\lambda_{n}\right) /\|\psi\|^{2}
$$

again assuming the requisite convergences. Whether these averages are real depends on both $f$ and $\left\{\lambda_{n}\right\}$. Also, for any given $L$, the action of the operator on $\psi_{\text {dual }}(z)$ is not necessarily obvious from its action on $\psi$. Similar statements apply if we interchange the rôles of $\psi$ and $\psi_{\text {dual }}$.

For any biorthogonal system, it is always possible, at least formally, to express the norms (6) for functions in the span of $\left\{\psi_{j}\right\}$ as integrals of bilinears in $\psi$ and $\psi^{*}$, where the construction is bilocal, in the general situation. If we build a kernel from the dual basis functions as follows

$$
K(\bar{w}, z) \equiv \sum_{n} \overline{\chi_{n}(w)} \chi_{n}(z)
$$


once again assuming the requisite convergences, we then have

$$
\begin{aligned}
\psi_{\text {dual }}(z) & =\int \overline{\psi(w)} K(\bar{w}, z) d \bar{w} \\
\|\psi\|^{2} & =\iint \overline{\psi(w)} K(\bar{w}, z) \psi(z) d \bar{w} d z
\end{aligned}
$$

where the contour for $\bar{w}$ is the complex conjugate of that for $z$. Similar results follow, at least formally, from constructing a dual bilocal kernel and integrating this with the associated dual functions.

$$
\begin{aligned}
J(\bar{w}, z) & \equiv \sum_{n} \overline{\psi_{n}(w)} \psi_{n}(z) \\
\overline{\psi(w)} & =\int J(\bar{w}, z) \psi_{\text {dual }}(z) d z \\
\|\psi\|^{2} & =\iint \overline{\psi_{\text {dual }}(w)} J(\bar{w}, z) \psi_{\text {dual }}(z) d \bar{w} d z
\end{aligned}
$$

Also, note that the convergences of the sums for $J$ and $K$ can be affected by making compensating changes in the individual normalizations of the elements in the biorthogonal system: $\left\{\psi_{j}, \chi_{k}\right\} \rightarrow\left\{Z_{j} \psi_{j}, \chi_{k} / Z_{k}\right\}$. By suitable choice of the $Z \mathrm{~s}$, the sum for one of the kernels can be made to converge uniformly, and rapidly. In the process, the sum for the other kernel may well become a divergent (perhaps asymptotic) series.

Formal expressions for other kernels follow immediately from acting with various operators on the $\left\{\psi_{j}\right\}$ or $\left\{\chi_{k}\right\}$ functions, or their conjugates, under the sums. For instance, returning to the previous eigenvalue example,

$$
J_{f(L)}(\bar{w}, z) \equiv \sum_{n} \overline{\psi_{n}(w)} f(L) \psi_{n}(z)=\sum_{n} \overline{\psi_{n}(w)} f\left(\lambda_{n}\right) \psi_{n}(z)
$$

which allows writing the average (17) as

$$
\langle f\rangle_{\psi}=\frac{1}{\|\psi\|^{2}} \iint \overline{\psi_{\text {dual }}(w)} J_{f(L)}(\bar{w}, z) \psi_{\text {dual }}(z) d \bar{w} d z
$$

In the $J_{f(L)}(\bar{w}, z)$ form, hermitian kernels leading to real averages are easily recognized when $f\left(\lambda_{n}\right)=\overline{f\left(\lambda_{n}\right)}$ for all $n$. Similar statements hold for kernels $K_{g\left(L_{\mathrm{dual}}\right)}$ built of bilinears in the dual functions, when the $\chi_{n}(z)$ are eigenfunctions of operators $L_{\mathrm{dual}}$.

Examples For an elementary example, let $\left\{\psi_{k}\right\}=\left\{z^{k} \mid k=0,1,2, \cdots\right\}$, i.e. the usual basis for analytic functions near the origin, and take the contour to be any simple closed curve surrounding $z=0$. Then $\chi_{k}(z)=z^{-k-1} / 2 \pi i$, for $k=0,1,2, \cdots$. If the contour is a circle of fixed radius $R$, with $z=R \exp (-i \theta)$, and if for convenience we incorporate various factors into a measure, $d \mu=-d z / 2 \pi i z=d \theta / 2 \pi$, then the previous sequence $\left\{z^{k}\right\}$ becomes the familiar set of left-moving eigenfunctions of $p_{\theta}=-i \partial / \partial \theta,\left\{\psi_{k}(\theta)\right\}=$ $\left\{R^{k} \exp (-i k \theta) \mid k=0,1, \cdots\right\}$, and the dual functions become (upon removal of $1 / z$ ) the right-movers, $\left\{\chi_{k}(\theta)\right\}=\left\{\frac{\exp (i k \theta)}{R^{k}} \mid k=0,1, \cdots\right\}$, so that

$$
\frac{1}{2 \pi} \int_{0}^{2 \pi} \chi_{k}(\theta) \psi_{j}(\theta) d \theta=\delta_{k j}
$$

But even in this familiar example, as defined, $\chi_{k}(\theta) \neq \psi_{k}^{*}(\theta)$ unless $R=1$. The bilocal norm kernels are easily constructed for this elementary case: $J(\bar{\theta}, \theta)=\sum_{n=0}^{\infty} R^{2 n} \exp i n(\bar{\theta}-\theta)=1 /\left(1-R^{2} \exp i(\bar{\theta}-\theta)\right)$; $K(\bar{\theta}, \theta)=\sum_{n=0}^{\infty} R^{-2 n} \exp i n(\theta-\bar{\theta})=R^{2} /\left(R^{2}-\exp i(\theta-\bar{\theta})\right)$. As functions of $R$, the complementary convergence properties of $J$ and $K$ are manifest.

A more interesting example was discovered by Neumann [70] in his work on Bessel functions (see $\S 9.1-17$ in [82]). This example is relevant to our discussion of PT invariant Hamiltonians to follow. For all analytic Bessel functions of non-negative integer index

$$
J_{n}(z)=\left(\frac{z}{2}\right)^{n} \sum_{k=0}^{\infty} \frac{(-1)^{k}}{k !(k+n) !}\left(\frac{z}{2}\right)^{2 k}
$$


there are corresponding associated Neumann polynomials $\left\{A_{n}\right\}$ in powers of $1 / z$ that are dual to $\left\{J_{n}\right\}$ on any contour enclosing the origin. These are given by

$$
A_{0}(z)=1, \quad A_{1}(z)=\frac{2}{z}, \quad A_{n \geq 2}(z)=n\left(\frac{2}{z}\right)^{n} \sum_{k=0}^{\lfloor n / 2\rfloor} \frac{(n-k-1) !}{k !}\left(\frac{z}{2}\right)^{2 k}
$$

For convenience we have modified the usual form of the associated polynomials, $O_{n}$ (as given in 70, 82, 11), and defined $A_{n}(z)=\varepsilon_{n} z O_{n}(z)$ where $\varepsilon_{0}=1$ and $\varepsilon_{n}=2$ for $n \neq 0$. Upon integrating counterclockwise once around $z=0$,

$$
\frac{1}{2 \pi i} \oint \frac{d z}{z} A_{j}(z) J_{k}(z)=\delta_{j k}
$$

so that $\left\{J_{n}(z)\right\}$ and $\left\{A_{n}(z)\right\}$ constitute a biorthogonal system on the complex plane in the same sense as $\left\{z^{n}\right\}$ and $\left\{z^{-n}\right\}$, but with the very significant difference that $A_{n}(z) \neq \overline{J_{n}(z)}$ even when $z$ is on the unit circle.

There are invertible maps between these two biorthogonal systems, $\left\{z^{j}, z^{-k}\right\} \longleftrightarrow\left\{J_{j}(z), A_{k}(z)\right\}$, about which we will say more later. The existence of such maps can be inferred from (17) and (18) as well as the reversions of those series to obtain all powers of $z$.

$$
\begin{gathered}
1=J_{0}(z)+2 \sum_{n=1}^{\infty} J_{2 n}(z), \quad z^{k}=2^{k} \sum_{n=0}^{\infty} \frac{(k+2 n)(k+n-1) !}{n !} J_{k+2 n}(z) \quad \text { where } \quad k=1,2,3, \cdots \\
\frac{1}{z^{2 j}}=\frac{1}{2^{2 j}} \sum_{k=0}^{j} \frac{(-)^{j-k} A_{2 k}(z)}{(j-k) !(j+k) !}, \quad \frac{1}{z^{2 j-1}}=\frac{1}{2^{2 j-1}} \sum_{k=1}^{j} \frac{(-)^{j-k} A_{2 k-1}(z)}{(j-k) !(j-1+k) !}
\end{gathered}
$$

Some bilocal kernels in closed form Neumann found that the Cauchy kernel is given by $(\underline{82}, \S 9.1)$

$$
\frac{1}{w-z}=\frac{1}{w} \sum_{n=0}^{\infty} A_{n}(w) J_{n}(z)
$$

When rewritten as

$$
\text { Id }(w, z) \equiv \frac{z}{z-w}=\sum_{n=0}^{\infty} J_{n}(w) A_{n}(z)
$$

this kernel acts as the identity when integrated on the space of Bessel functions (or alternatively on Neumann polynomials) provided the contours of integration are appropriately chosen to handle the singularity at $w=z$.

$$
J_{k}(w)=\frac{1}{2 \pi i} \oint_{|z|>|w|} \frac{d z}{z} \operatorname{Id}(w, z) J_{k}(z), \quad A_{k}(z)=\frac{1}{2 \pi i} \oint_{|w|<|z|} \frac{d w}{w} A_{k}(w) \operatorname{Id}(w, z)
$$

Acting with Bessel's operator on this kernel leads in turn to an example of a bilocal eigenoperator kernel.

$$
\begin{aligned}
& \mathbf{H}(w, z) \equiv\left(w^{2} \frac{d^{2}}{d w^{2}}+w \frac{d}{d w}+w^{2}\right) \operatorname{Id}(w, z)=\frac{2 w^{2} z}{(z-w)^{3}}+\frac{w z}{(z-w)^{2}}+\frac{w^{2} z}{z-w} \\
&=\sum_{n=0}^{\infty} n^{2} J_{n}(w) A_{n}(z) \\
& k^{2} J_{k}(w)=\frac{1}{2 \pi i} \oint_{|z|>|w|} \frac{d z}{z} \mathbf{H}(w, z) J_{k}(z), \quad k^{2} A_{k}(z)=\frac{1}{2 \pi i} \oint_{|w|<|z|} \frac{d w}{w} A_{k}(w) \mathbf{H}(w, z)
\end{aligned}
$$

Neumann also showed that $(\underline{82}, \S 11.1-2)$

$$
J_{0}\left(\sqrt{w^{2}+z^{2}-2 w z \cos \phi}\right)=\sum_{n=-\infty}^{\infty} J_{n}(w) J_{n}(z) \cos (n \phi)
$$


where $J_{-n}(z)=(-1)^{n} J_{n}(z)$ and $J_{0}(s)=\sum_{k=0}^{\infty} \frac{(-1)^{k}}{(k !)^{2}}\left(\frac{s^{2}}{4}\right)^{k}$. From 27) we obtain an explicit and closed form for the dual kernel used in the construction of the bilocal norm for this biorthogonal system, as in (11).

$$
J(\bar{w}, z)=\sum_{n=0}^{\infty} J_{n}(\bar{w}) J_{n}(z)=\frac{1}{2}\left(J_{0}(\bar{w}-z)+J_{0}(\bar{w}) J_{0}(z)\right)
$$

With the normalizations chosen, the sum for the other kernel $K(\bar{w}, z)=\sum_{n=0}^{\infty} A_{n}(\bar{w}) A_{n}(z)$ is an asymptotic series. However, as discussed earlier in a general context, the $J(\bar{w}, z)$ and $K(\bar{w}, z)$ series convergence properties can be reversed by making compensating changes in the normalizations: $\left\{J_{j}, A_{k}\right\} \rightarrow\left\{Z_{j} J_{j}, A_{k} / Z_{k}\right\}$. We will give other examples of kernels, in closed form, when we discuss the corresponding quantum systems below.

Generalizations The $\left\{J_{n}(z), A_{n}(z)\right\}$ biorthogonal system has been generalized by Gegenbauer to other situations involving Bessel functions with non-integral indices and their associated polynomials (see $§ 9.2$ in [82]). This is relevant to our discussion, given below, of some simple magnetic field effects. Extensions to confluent hypergeometric functions ${ }_{1} F_{1}$ have been proposed by Erdélyi 38. These are relevant to $H=(p+\nu)^{2}+\mu_{1} \exp (i x)+\mu_{2} \exp (2 i x)$ for arbitrary $\mu_{1}$ and $\mu_{2}$, as also discussed below. We will further generalize the polynomials to all systems governed by Hamiltonians of the form (11). But, before fleshing out the biorthogonal structure in the various soluble quantum mechanical models of interest to us, we give a survey of the classical dynamics for the first such model.

\section{Classical dynamics for $V(x)=\exp (2 i x)$}

Consider the Hamiltonian

$$
H=p^{2}+m^{2} e^{2 i x}
$$

where $m$ is real. This is a complex form of the well-studied Liouville dynamics [15, 69. The coefficient $m^{2}$ of the exponential has to be real for the Hamiltonian to be PT symmetric. That is to say, $H$ is invariant under $p \rightarrow p, x \rightarrow-x$, and complex conjugation. Or at least, that is the case when we restrict to real $x$ and real $p$. These transformations are inspired by the conventional PT transformations of quantum mechanics, for wave functions $\psi(x, t)$ that depend on real $x$ and $t$. The phase of $m^{2}$ can be changed to any arbitrary value just by making a real shift in $x$, but only shifts $\Delta x=n \pi / 2$ for integer $n$ will preserve PT invariance. Still, we may say all values of $m$, even if complex, give theories translationally equivalent to PT symmetric models.

While this has to be the simplest periodic complex potential example one can imagine, nevertheless, it is not obvious a priori how or if the dynamics is consistent, nor is it obvious what connections exist between a classical system described by (29) and any quantum deformation of that dynamics. A goal of this paper is to examine this consistency in some detail, and to discuss these connections.

While simple, this is still an interesting dynamical system. However, there is some ambiguity about what $H$ represents when $p$ and $x$ are complex. Following Bender et al. [6], we choose to continue the model into the complex plane simply by complexification of the usual form of Hamilton's equations of motion, without complex conjugation of any of the variables appearing in those equations. Thus we take (29) and

$$
\frac{d x(t)}{d t}=\frac{\partial H}{\partial p}=2 p(t), \quad \frac{d p(t)}{d t}=-\frac{\partial H}{\partial x}=-2 i m^{2} e^{2 i x(t)}
$$

to hold for all complex $x$ and $p$. With this definition of the continuation, the complex energy is conserved $d H / d t=0$. So under this complexification procedure there are in fact two conserved real quantities, $\operatorname{Re} H$ and $\operatorname{Im} H$.

Complex energy conservation allows reduction to a single first order equation

$$
\frac{d x(t)}{d t}=2 p(t)= \pm 2 \sqrt{E-m^{2} e^{2 i x(t)}}
$$


with integration in terms of elementary functions. This yields the classical motion.

$$
x(t)=\frac{1}{2 i} \ln \left(\frac{E / m^{2}}{\cosh ^{2}\left(\mp 2 i \sqrt{E} t+\operatorname{arctanh} \frac{\sqrt{E-m^{2} e^{2 i x(0)}}}{\sqrt{E}}\right)}\right)
$$

Sign flips on the RHSs of (31) and (32) occur at complex turning points, as given by $\operatorname{Re} \sqrt{E-m^{2} e^{2 i x(t)}}=$ $0=\operatorname{Im} \sqrt{E-m^{2} e^{2 i x(t)}}$. If no $\operatorname{Re} \sqrt{E-m^{2} e^{2 i x(t)}}=0$ points are encountered, upon taking the upper signs in (31) and (32), the motion is a continuous but varying progression towards positive Re $x$ with periodic oscillations in $\operatorname{Im} x$. We plot a representative $E>0$ example $(E=1 / 4, x(0)=1, \operatorname{Re} p(0)>0)$ showing real and imaginary parts of both $x$ and $p$ as parametric functions of $t$.

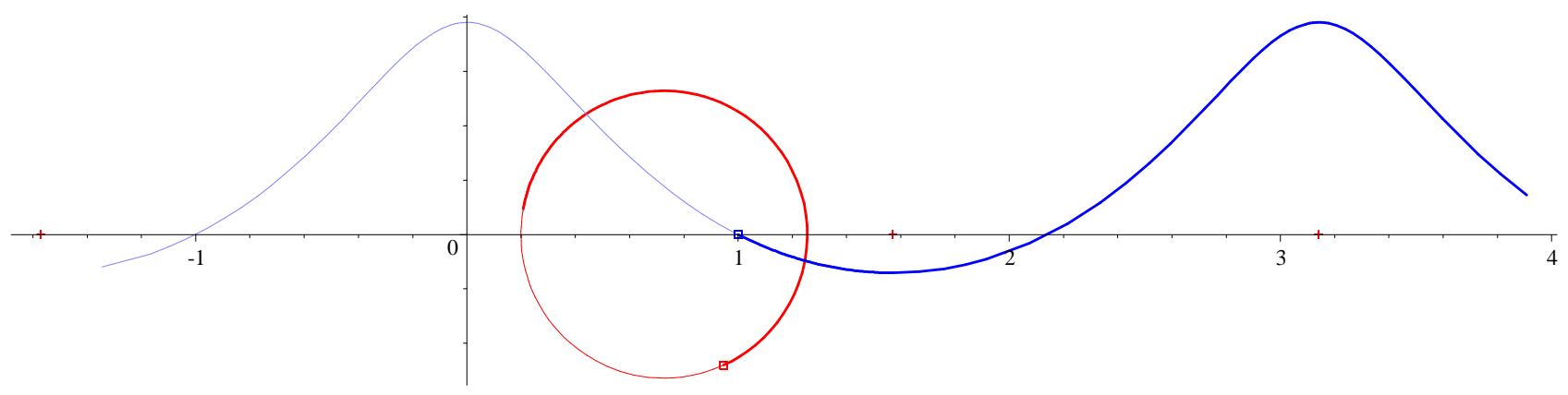

$(\operatorname{Re} x, \operatorname{Im} x)$ in blue and $(\operatorname{Re} p, \operatorname{Im} p)$ in red, plotted parametrically for $E=1 / 4$

In this and all other plots to follow, we have set $m=1$. Heavier/lighter curves indicate forward/backward evolution in $t$. Initial points are indicated by small boxes in the Figure.

The momentum trajectory in the Figure is as simple as it looks: A circle. For all real $E>0$ and any initial $x(0)$ the momentum $p(t)$ evolves around a complex circle centered on the real $p$ axis. This can be shown by inserting (32) into (31) and manipulating the result to obtain

$$
p(t)= \pm \sqrt{E}\left(\frac{a-e^{ \pm 4 i \sqrt{E} t}}{a+e^{ \pm 4 i \sqrt{E} t}}\right)
$$

where we have defined the complex amplitude

$$
a \equiv \frac{E}{m^{2}}\left(1+\sqrt{1-\frac{m^{2}}{E} e^{2 i x(0)}}\right)^{2} e^{-2 i x(0)}
$$

Hence $a$ depends on the initial data but not on $t$. In this form, $a$ and $p$ have transparent high energy limits. From (33) it is straightforward to establish by direct calculation for real $E>0$ that $p(t)$ evolves around a circle in the complex plane, of radius $\frac{2|a|}{|a|^{2}-1} \sqrt{E}$, whose center is on the real axis at $\pm \frac{|a|^{2}+1}{|a|^{2}-1} \sqrt{E}$. It also follows from (33) that the momentum's angular velocity around the circle is not constant, but varies. In addition, for real $E>0$ a turning point is encountered if and only if $|a|=1$, implying the $p$ circle has infinite radius and degenerates into a vertical straight line in the complex plane, as described by purely imaginary momentum trajectories $p(t)=-i \sqrt{E} \tan \left(2 \sqrt{E} t \mp \frac{1}{2} \arg a\right)$. Correspondingly, the $x$ trajectory for $|a|=1$ is along a vertical line in the complex plane, with fixed Re $x$. For example, this can occur when $x(0)=N \pi$ and $E=m^{2}$, in which cases the $x$ trajectory "bounces off" the real axis at $t=0$.

The $E=0$ motion is somewhat simpler, but still has interesting structure, and is given as follows.

$$
x_{E=0}(t)=i \ln \left(e^{-i x(0)} \pm 2 m t\right), \quad \frac{d x_{E=0}(t)}{d t}=\frac{ \pm 2 i m}{e^{-i x(0)} \pm 2 m t}
$$


Again, the momentum evolves along a circle of finite radius in the complex plane, approaching the origin as a limit, except in the special situations $x(0)=N \pi$, which correspond to circles of infinite radius. In these special cases, the particle stays at $\operatorname{Re} x_{E=0}=N \pi$ for all $t$, but moves in the imaginary direction, with

$$
\begin{aligned}
\left.\operatorname{Im} x_{E=0}(t)\right|_{\operatorname{Re} x_{E=0}=N \pi} & =\frac{1}{2} \ln \left(1+4 m^{2} t^{2} \pm 4 m t(-)^{N}\right) \\
& =\ln (1 \pm 2 m t)
\end{aligned}
$$

and with \pm signs depending on $N$ as well as the choice of root for $p_{E=0}$. So with the \pm as exhibited in (36), $x_{E=0}$ goes to $x=+i \infty$ as $t \rightarrow \pm \infty$ and goes to $x=-i \infty$ as $t \rightarrow \mp \frac{1}{2 m}$. In fact, no matter what $x(0)$ is, the $E=0$ solution always has an imaginary part that behaves as $\operatorname{Im} x_{E=0}(t) \underset{t \rightarrow \pm \infty}{ } \ln \left(t^{2}\right)$ for at least one choice of sign.

We plot parametrically some $E=0$ examples, for various initial $x(0)$, showing real and imaginary parts of $x(t)$ and $p(t)$ for forward/backward evolution. The initial positions are $x(0)=\pi / 2$ (blue), 2.5 (green), 2.8 (black), and $\pi$ (red), as indicated by small boxes in the Figure on the left. Corresponding initial values for $p$ are also indicated by small boxes in the Figure on the right.
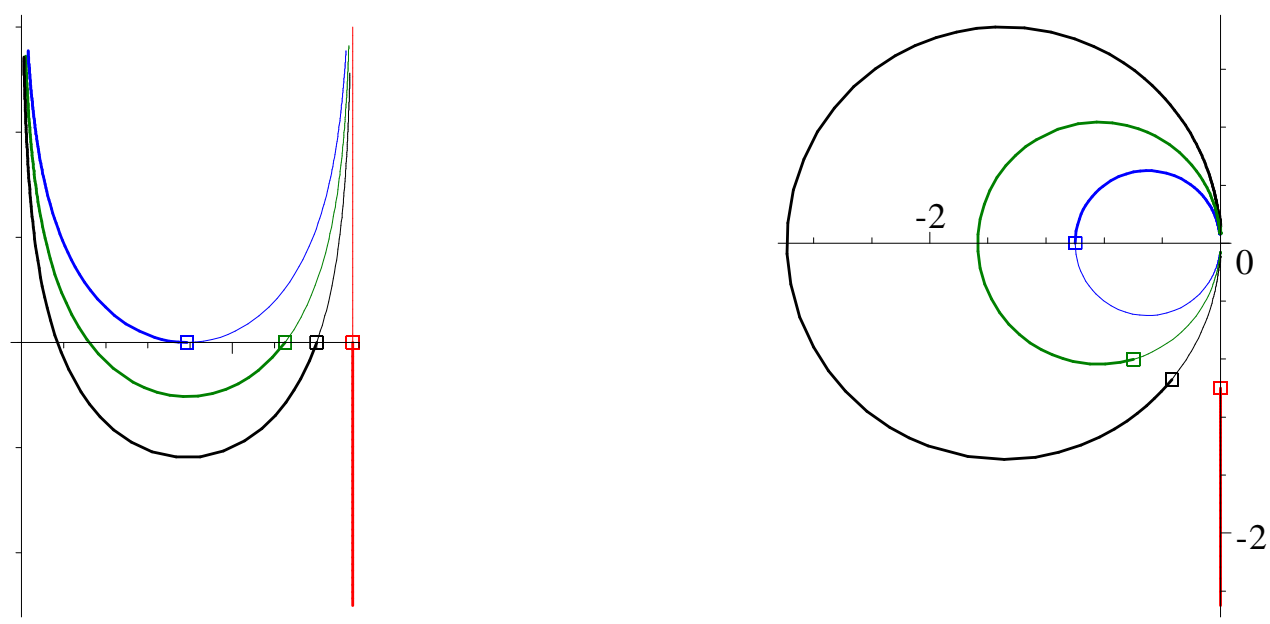

$(\operatorname{Re} x, \operatorname{Im} x)$ for $E=0$ and various initial positions $\quad(\operatorname{Re} p, \operatorname{Im} p)$ for $E=0$ and various initial momenta

Canonical transformations Many of the classical properties are more easily understood upon taking note of the following fact. There exist generating functions for canonical transformations from Liouville to free particle dynamics [17, with free variables $\theta$ and $p_{\theta}$. However, in the present context, both free particle and Liouville dynamics must be complexified, in general. One such transformation is given by

$$
F=m e^{i x} \sin \theta, \quad p \equiv \frac{\partial}{\partial x} F=i m e^{i x} \sin \theta, \quad p_{\theta} \equiv-\frac{\partial}{\partial \theta} F=-m e^{i x} \cos \theta
$$

The free particle's momentum is conserved, $p_{\theta}= \pm \sqrt{E}$, since under the transformation

$$
H=p^{2}+m^{2} e^{2 i x}=p_{\theta}^{2}=H_{\text {free }}
$$

For real $E>0, p_{\theta}$ is also real. Although this would in general require complex $x, p$, and $\theta$, as follows from the transformation. Separating out the $(x, p)$ and $\left(\theta, p_{\theta}\right)$ variables in (37), and expressing one set in terms of the other, leads to

$$
\begin{aligned}
& x=-i \ln \left(\frac{p_{\theta}}{-m \cos \theta}\right), \quad p=-i p_{\theta} \tan \theta \\
& \theta=\arcsin \left(-i e^{-i x} p / m\right), \quad p_{\theta}=-m e^{i x} \cos \left(\arcsin \left(-i e^{-i x} p / m\right)\right)
\end{aligned}
$$


where some care is needed if cuts are encountered. These relations lead most easily to the result that $p(t)$ lies on a complex circle, for real $E>0$, as a simple consequence of $\theta(t)=\theta(0)+2 p_{\theta} t$, where $\operatorname{Im} \theta(t)=\operatorname{Im} \theta(0)$ is an invariant for real $E>0$. Comparing (39) to (33), we see that $a=\exp (-2 i \theta(0))$, so $|a|=\exp (2 \operatorname{Im} \theta(0))$. Thus, $x(t)$ turning points are encountered for real $E>0$ if and only if $\operatorname{Im} \theta=0$ on the corresponding free particle trajectories.

Actually, this canonical transformation works no matter what the energy is, even if it is complex, in which case the classical trajectories can have an elegant appearance. The Figure below shows a momentum trajectory for $x(0)=1$ and $E=e^{i}$. The flow is between symmetrical complex fixed points at $p_{ \pm}= \pm e^{i / 2}$.

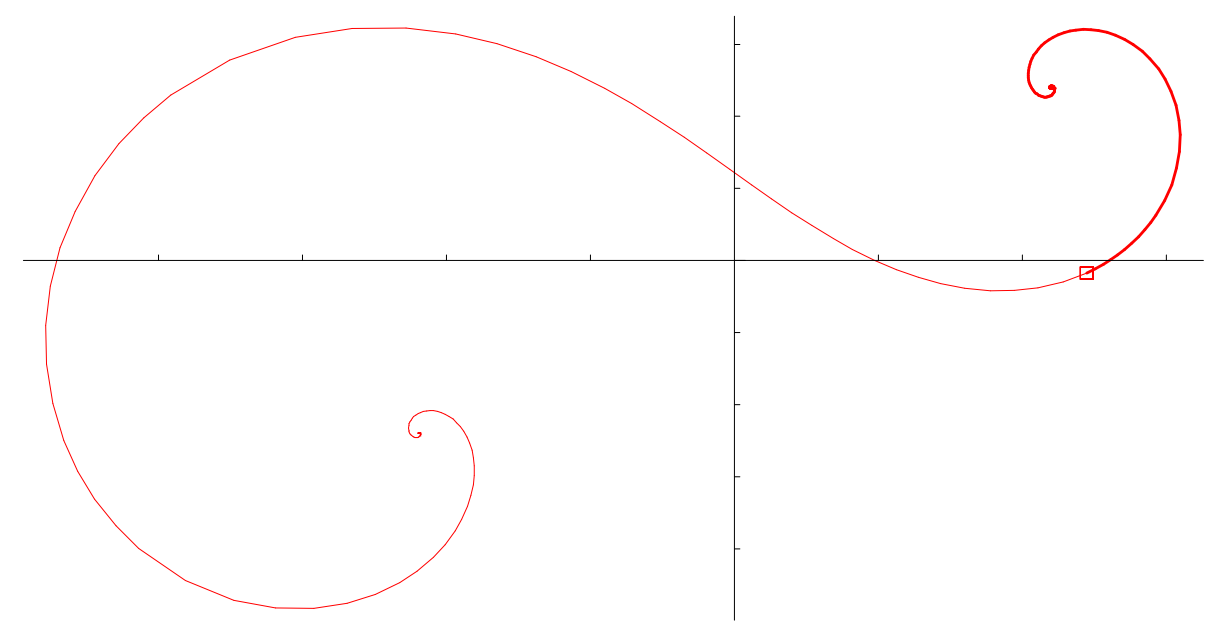

The choice $E=e^{2 i}$ would give a more symmetrical trajectory, with $p(t)=-p(-t)$, and with a turning point. Of course, as indicated by the explicit transformation (39), complex energy would require extending the free particle trajectories into both the complex $\theta$ and the complex $p_{\theta}$ planes.

The classical trajectories and the classical canonical transformation which we have just described will appear explicitly in the quantum mechanics of the model, to be discussed in the next section, where they play pivotal roles in canonical integral representations of the wave functions and their duals. But before ending our discussion of classical dynamics and turning our attention to quantization, some comments on the canonical structure of this system would seem to be in order. Indeed, this issue is brought into particular focus by our previous remarks concerning classical canonical transformations.

We might have proceeded as in the usual case of a particle on the plane, and assumed the real and imaginary components of $x=\operatorname{Re} x+i \operatorname{Im} x$ and $p=\operatorname{Re} p+i \operatorname{Im} p$ are independently canonical, up to some overall "isotropic" scale $\sigma$, with Poisson brackets $(\mathrm{PBs})$ given by $[\operatorname{Re} x, \operatorname{Re} p]_{\mathrm{PB}}=\sigma,[\operatorname{Im} x, \operatorname{Im} p]_{\mathrm{PB}}=\sigma$, $[\operatorname{Re} x, \operatorname{Im} x]_{\mathrm{PB}}=0,[\operatorname{Re} x, \operatorname{Im} p]_{\mathrm{PB}}=0$, etc. Had we done so, we would then have had for any value of $\sigma$ the brackets $[x, p]_{\mathrm{PB}}=0,[x, H]_{\mathrm{PB}}=0,[p, H]_{\mathrm{PB}}=0$. However, this is not the canonical structure that leads to Hamiltonian dynamics as given in (30), nor is it the structure that underlies the classical canonical transformation given in (37). To obtain non-trivial equations of motion and other canonical transformations of variables, in this isotropic Poisson bracket approach, it would have been necessary to also conjugate some of the dynamical variables appearing in $H$ and the generating function $F$. But we chose not to do this.

On the other hand, if we were to suppose the brackets are different for the real and imaginary parts, and modify them to be anisotropic brackets (ABs) as

$$
[\operatorname{Re} x, \operatorname{Re} p]_{\mathrm{AB}}=\sigma+\cos ^{2} \zeta, \quad[\operatorname{Im} x, \operatorname{Im} p]_{\mathrm{AB}}=\sigma-\sin ^{2} \zeta
$$

$[\operatorname{Re} x, \operatorname{Im} x]_{\mathrm{AB}}=0,[\operatorname{Re} x, \operatorname{Im} p]_{\mathrm{AB}}=0$, etc., then for any values of $\sigma$ and $\zeta$ we would have

$$
[x, p]_{\mathrm{AB}}=1, \quad[x, H]_{\mathrm{AB}}=2 p, \quad[p, H]_{\mathrm{AB}}=-2 i m^{2} e^{2 i x}
$$

This is exactly the dynamics specified in (30) if we identify $d f(x, p) / d t=[f(x, p), H]_{\mathrm{AB}}$, in the usual way. If we were to go further and impose the additional condition that the bracket of $x$ with the complex 
conjugate of $p$ vanish, then we would be led to $2 \sigma+\cos 2 \zeta=0$, hence $\sigma+\cos ^{2} \zeta=1 / 2$ and $\sigma-\sin ^{2} \zeta=-1 / 2$. This would give the most symmetrical form for the anisotropic brackets. For this choice the real and imaginary components would have brackets differing by a naive PT transformation, i.e. just a sign flip: $[\operatorname{Re} x, \operatorname{Re} p]_{\mathrm{AB}}=1 / 2,[\operatorname{Im} x, \operatorname{Im} p]_{\mathrm{AB}}=-1 / 2$. This is what we have done.

We will briefly return to this issue at the end of the next section, in a quantum phase-space framework, by considering deformations of the anisotropic brackets.

\section{Quantum mechanics for $V(x)=\exp (2 i x)$}

Reconsider the PT symmetric Hamiltonian, (29), only now in a quantum setting. Again, granted that PT symmetric quantum theories make sense, this has to be the simplest periodic quantum potential problem one can imagine. ${ }^{1}$ The corresponding Schrödinger energy eigenvalue equation is

$$
\left(-\frac{\partial^{2}}{\partial x^{2}}+m^{2} e^{2 i x}\right) \psi_{E}=E \psi_{E}
$$

Since the potential is periodic, with period $\pi$, Floquet's theorem assures us that we can always find a solution of the form

$$
\psi(x)=e^{i \mu x} \phi(x)
$$

where $\phi(x+\pi)=\phi(x)$. That is to say, $\phi(x)$ has the form $\phi(x)=\sum_{n \in \mathbb{Z}} c_{n} e^{2 i n x}$. These features are easily borne out, since the equation can be solved exactly. Change variables to

$$
z=m e^{i x}
$$

Then $\frac{\partial}{\partial x}=i z \frac{\partial}{\partial z}$ and (42) becomes

$$
\left(z^{2} \frac{\partial^{2}}{\partial z^{2}}+z \frac{\partial}{\partial z}+z^{2}-E\right) \psi(z)=0
$$

This of course is Bessel's equation, albeit in the perhaps unfamiliar situation where the variable $z / m$ is on the unit circle. Series solutions are explicitly given by

$$
J_{ \pm \sqrt{E}}\left(m e^{i x}\right)=\left(\frac{m}{2} e^{i x}\right)^{ \pm \sqrt{E}} \sum_{n=0}^{\infty} \frac{\left(-m^{2} / 4\right)^{n}}{n ! \Gamma(1+n \pm \sqrt{E})} e^{2 i n x}
$$

and exhibit features in accord with Floquet's theorem. For completeness, we also recall the elegant contour integral representation of Schläfli and Sonine $(82 \S 6.2)$ which is valid for all $\sqrt{E}$ and $x$.

$$
J_{ \pm \sqrt{E}}\left(m e^{i x}\right)=\left(\frac{m}{2} e^{i x}\right)^{ \pm \sqrt{E}} \frac{1}{2 \pi i} \int_{-\infty}^{(0+)} w^{\mp \sqrt{E}-1} \exp \left(w-\frac{m^{2} e^{2 i x}}{4 w}\right) d w
$$

The contour begins at $-\infty$, with $\arg w=-\pi$, proceeds below the real $w$ axis towards the origin, loops in the positive, counterclockwise sense around the origin (hence the $(0+)$ notation), and then continues above the real $w$ axis back to $-\infty$, with $\arg w=+\pi$.

The two choices of sign on the index of the Bessel function give independent solutions, so long as $\sqrt{E}$ is not an integer. The prefactor in (46) or (47) is just a free plane wave, for all real $E \geq 0$. The infinite sum (or Sonine's integral) provides periodic modulations and additional phases to the plane wave. Negative or complex $E$ would give for the solutions either an exponential blow-up for $x$ on the real line, or else non-periodic behavior for $x \in[0,2 \pi N]$, where in the latter case periodicity requires $\sqrt{E}=k / N$ for some integer $k$. For these reasons negative or complex $E$ is always ruled to be inadmissible. In this regard, we

\footnotetext{
${ }^{1}$ This example is mentioned in passing by Bender et al. 8, without much discussion. It is considered in more detail in 20. Moreover, the model has been discussed extensively in the mathematical literature, at least as early as the 1950s

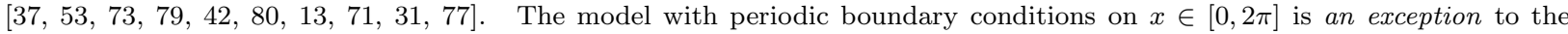
method used by Bender et al. to construct the dual space. We will discuss this fully in the following.
} 
emphasize that our discussion applies to $x$ on the real line, or else to $z=m e^{i x}$ lying on a closed contour surrounding the origin. For energy spectra corresponding to boundary conditions on other contours in the complex plane, see $\S 5$ in [20].

So, the solutions of (42) for all real $E \geq 0$ are (recall $J_{n}(z)=(-1)^{n} J_{-n}(z)$ for integer index)

$$
\begin{aligned}
& \psi_{E}^{+}(x)=\frac{1}{2}\left(J_{\sqrt{E}}\left(m e^{i x}\right)+J_{-\sqrt{E}}\left(m e^{i x}\right)\right) \underset{\sqrt{E} \rightarrow n \in \mathbb{N}}{\longrightarrow}\left\{\begin{array}{cc}
J_{n}\left(m e^{i x}\right) & \text { for } n \text { even } \\
0 & \text { for } n \text { odd }
\end{array}\right. \\
& \psi_{E}^{-}(x)=\frac{1}{2}\left(J_{\sqrt{E}}\left(m e^{i x}\right)-J_{-\sqrt{E}}\left(m e^{i x}\right)\right) \underset{\sqrt{E} \rightarrow n \in \mathbb{N}}{\longrightarrow}\left\{\begin{array}{cc}
0 & \text { for } n \text { even } \\
J_{n}\left(m e^{i x}\right) & \text { for } n \text { odd }
\end{array}\right.
\end{aligned}
$$

We have chosen linear combinations of $J_{ \pm \sqrt{E}}$ for later convenience. We have also chosen the phases of the eigenstates so that $\mathcal{P} \mathcal{T} \psi_{E}^{ \pm}(x)=\psi_{E}^{ \pm}(x)$. When $E=0$, since $\psi_{E=0}^{-} \equiv 0$, we have a non-degenerate ground state, $\psi_{E=0}^{+}(x)=J_{0}\left(m e^{i x}\right)$. This is unlike the case of real Liouville theory, for which there is no ground state 32 .

In fact, note the lack of degeneracy whenever $\sqrt{E}$ is any integer. If $\sqrt{E}=n \geq 0$, the two independent solutions of (45) are usually taken to be $J_{\sqrt{E}}(z)$ and $\lim _{\sqrt{E} \rightarrow n} Y_{\sqrt{E}}(z)=\lim _{\sqrt{E} \rightarrow n} \frac{J_{\sqrt{E}}(z) \cos (\pi \sqrt{E})-J_{-\sqrt{E}}(z)}{\sin (\pi \sqrt{E})}$, the latter being well-defined even as $\sqrt{E} \rightarrow$ integer. But, although $Y_{n}\left(m e^{i x}\right)$ is finite for real $x$, nevertheless it can still be ruled out as an admissible solution because $Y_{n}$ involves a logarithm multiplying a periodic function. Hence it has a periodically modulated term with an envelope linear in $x$, so it is unbounded for $-\infty<x<\infty$. Alternatively, $Y_{n}\left(m e^{i x}\right)$ is not a periodic function for $x \in[0,2 \pi]$. So $Y_{n}\left(m e^{i x}\right)$ is not an admissible solution on the domains of interest to us.

We plot $\left(\operatorname{Re} \psi_{E}(x), \operatorname{Im} \psi_{E}(x)\right)$ and $\left(\operatorname{Re} \psi_{E}^{2}(x), \operatorname{Im} \psi_{E}^{2}(x)\right)$ parametrically, as functions of $x$, for two cases.

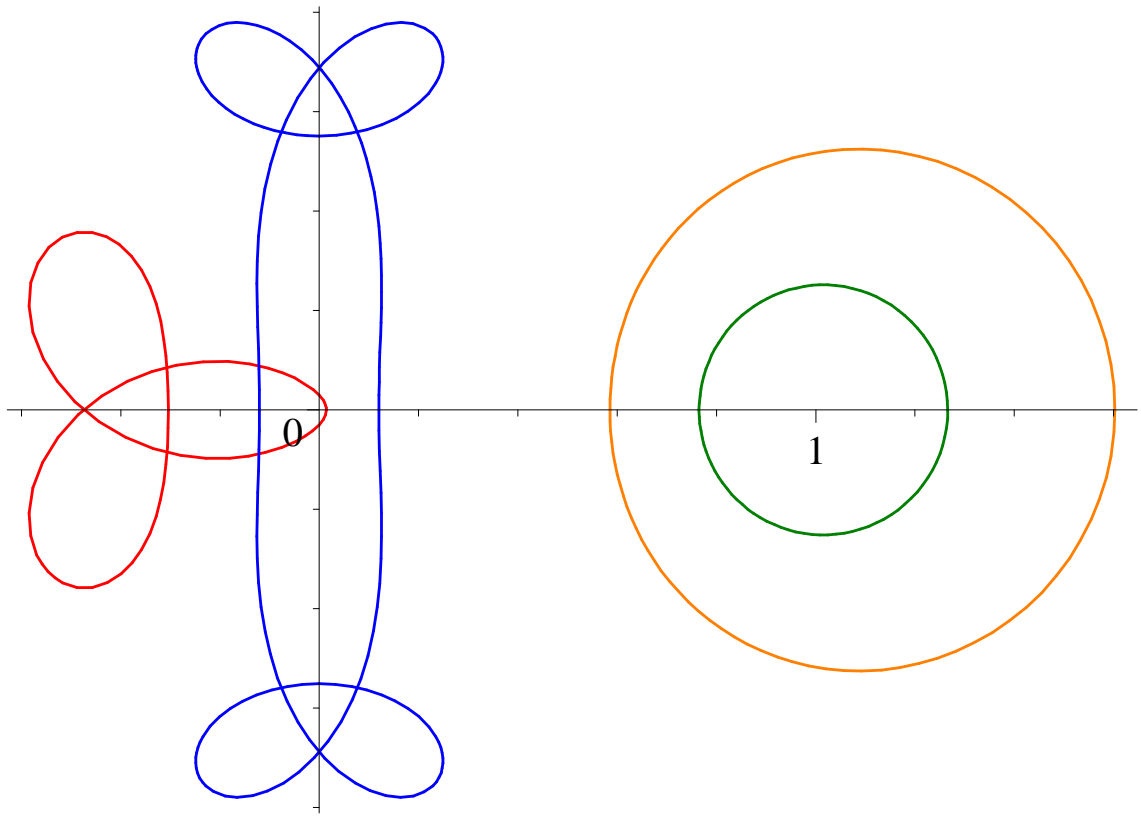

$\left(\operatorname{Re} \psi_{E}(x), \operatorname{Im} \psi_{E}\right)$ and $\left(\operatorname{Re} \psi_{E}^{2}, \operatorname{Im} \psi_{E}^{2}\right)$, for $E=0$ (green and orange) and for $E=1 / 4$ (blue and red)

In the case $E=0$, it is sufficient to take $x \in[0,2 \pi]$. In the case $E=1 / 4$, we have plotted $\psi_{1 / 4}^{-}$and $\left(\psi_{1 / 4}^{-}\right)^{2}$ for $x \in[-2 \pi, 2 \pi]$. The complete curve for the other wave function at this same energy, $\psi_{1 / 4}^{+}$, is 
obtained by rotating the complete $\psi_{1 / 4}^{-}$curve through $\pi / 2$ about the origin, even though point-by-point, $\psi_{1 / 4}^{+}(x) \neq e^{ \pm i \pi / 2} \psi_{1 / 4}^{-}(x)$. Similarly the complete $\left(\psi_{1 / 4}^{+}\right)^{2}$ curve is obtained by rotating $\left(\psi_{1 / 4}^{-}\right)^{2}$ by $\pi$.

From these plots it is clear that $\psi^{2}(x)$ is not a bona fide probability density since it is complex. It is also more or less clear from the symmetry of $\psi^{2}$ that $\int_{0}^{2 \pi}\left(\psi_{0}\right)^{2} d x$ is real and positive, while $\int_{-2 \pi}^{2 \pi}\left(\psi_{1 / 4}^{-}\right)^{2} d x$ is real and negative. These are the so-called PT inner products [5]. This leads to the problem of constructing the dual space for all the eigenfunctions.

When $\sqrt{E}$ is not an integer, the "CPT" construction of the dual space [5] goes through. This follows because

$$
\int_{-\infty}^{\infty} \psi_{E_{1}}^{ \pm}(x) \psi_{E_{2}}^{ \pm}(x) d x= \pm \delta\left(E_{1}-E_{2}\right) \times 2 \sin \left(\pi \sqrt{E_{1}}\right), \quad \int_{-\infty}^{\infty} \psi_{E_{1}}^{+}(x) \psi_{E_{2}}^{-}(x) d x=0
$$

and $\sin (\pi \sqrt{E})$ on the RHS does not vanish when $\sqrt{E}$ is not an integer. In this case, the dual functions can be identified with

$$
\chi_{E}^{ \pm}=\frac{ \pm 1}{2 \sin (\pi \sqrt{E})} \psi_{E}^{ \pm}(x)
$$

and exhibit the expected sign flips for alternating bands of energies. However, when $\sqrt{E}$ is an integer, $\sin (\pi \sqrt{E})$ vanishes, the PT symmetric eigenfunctions are self-orthogonal, and the CPT construction of the dual space fails. This happens for the invariant subspace of non-degenerate eigenfunctions which are periodic on $x \in[0,2 \pi]$. In the mathematical literature [42, 80, 77] these integer $\sqrt{E}$ are known as spectral singularities $^{2}$, a name seen to be appropriate from the behavior of the integrands for spectral resolutions of projectors.

$$
\mathbb{P}_{\left[E_{1}, E_{2}\right]}^{ \pm}(x, y)=\int_{E_{1}}^{E_{2}} \psi_{E}^{ \pm}(x) \psi_{E}^{ \pm}(y) \frac{ \pm 1}{2 \sin (\pi \sqrt{E})} d E
$$

Henceforth we consider only the subspace of $2 \pi$-periodic eigenfunctions.

A remarkable $2 \pi$-periodic biorthogonal system Elements of the dual space for the $2 \pi$-periodic eigenfunctions are given by the Neumann polynomials as presented earlier. The key orthogonality relation is now

$$
\frac{1}{2 \pi} \int_{0}^{2 \pi} A_{k}\left(m e^{i x}\right) J_{n}\left(m e^{i x}\right) d x=\delta_{k n}
$$

where $A_{-n}(z) \equiv(-1)^{n} A_{n}(z)$. This gives a remarkable biorthogonal system whose properties warrant more discussion. The CPT method of constructing the dual space, i.e. simply changing normalizations and phases of the PT transformed functions, does not provide a bi-orthonormalizable set of functions in this case, since

$$
\frac{1}{2 \pi} \int_{0}^{2 \pi} J_{k}\left(m e^{i x}\right) J_{n}\left(m e^{i x}\right) d x=\delta_{k 0} \delta_{n 0}
$$

This follows because the $J_{\mathrm{s}}$ are series in only positive powers of $e^{i x}$. So all the $2 \pi$-periodic energy eigenfunctions are self-orthogonal except for the ground state.

The effects of the Hamiltonian on the dual space The $A_{n}$ obey an inhomogeneous equation [82].

$$
-\frac{d^{2}}{d x^{2}} A_{n}\left(m e^{i x}\right)+\left(m^{2} e^{2 i x}-n^{2}\right) A_{n}\left(m e^{i x}\right)=\left\{\begin{array}{cl}
2 n m e^{i x} & \text { for } n \text { odd } \\
2 m^{2} e^{2 i x} & \text { for } n \text { even }
\end{array}\right.
$$

This leads to an interesting variant on the usual method of showing that eigenfunctions and their duals are orthogonal for different energies. Consider $\int_{0}^{2 \pi} A_{k}\left(m e^{i x}\right) H J_{n}\left(m e^{i x}\right) d x=n^{2} \int_{0}^{2 \pi} A_{k}\left(m e^{i x}\right) J_{n}\left(m e^{i x}\right) d x$.

\footnotetext{
${ }^{2}$ For other examples of this effect in PT symmetric theories, see 74 .
} 
Integrating by parts gives

$$
\begin{gathered}
\int_{0}^{2 \pi} A_{k}\left(m e^{i x}\right) H J_{n}\left(m e^{i x}\right) d x=\int_{0}^{2 \pi} J_{n}\left(m e^{i x}\right) H A_{k}\left(m e^{i x}\right) d x \\
=k^{2} \int_{0}^{2 \pi} J_{n}\left(m e^{i x}\right) A_{k}\left(m e^{i x}\right) d x+\left\{\begin{array}{cc}
2 k m \int_{0}^{2 \pi} e^{i x} J_{n}\left(m e^{i x}\right) d x & \text { for } k \text { odd } \\
2 m^{2} \int_{0}^{2 \pi} e^{2 i x} J_{n}\left(m e^{i x}\right) d x & \text { for } k \text { even }
\end{array}\right.
\end{gathered}
$$

There are no boundary contributions upon integrating by parts since all functions are $2 \pi$-periodic. Moreover, the inhomogeneous terms are orthogonal to the space of energy eigenfunctions. That is to say, $\int_{0}^{2 \pi} e^{i x} J_{n}\left(m e^{i x}\right) d x=0=\int_{0}^{2 \pi} e^{2 i x} J_{n}\left(m e^{i x}\right) d x$ since the $J_{\mathrm{S}}$ are series in only positive powers of $e^{i x}$. So even though (54) is inhomogeneous, we still arrive at the usual conclusion.

$$
\left(n^{2}-k^{2}\right) \int_{0}^{2 \pi} A_{k}\left(m e^{i x}\right) J_{n}\left(m e^{i x}\right) d x=0
$$

hence $\int_{0}^{2 \pi} A_{k}\left(m e^{i x}\right) J_{n}\left(m e^{i x}\right) d x=0$ if $k^{2} \neq n^{2}$. Recall that $A_{-n}(z) \equiv(-1)^{n} A_{n}(z)$.

Integral representations for $E=n^{2}$ and quantum equivalence to a free particle on a circle The $2 \pi$-periodic Bessel functions are in fact the canonical integral transforms of free plane waves on a circle, as constructed in this special situation just by exponentiating the classical generating function presented earlier in (37). Explicitly,

$$
J_{n}\left(m e^{i x}\right)=\frac{1}{2 \pi} \int_{0}^{2 \pi} e^{i m e^{i x} \sin \theta} e^{-i n \theta} d \theta, \quad n \in \mathbb{Z}
$$

The essence of the quantum equivalence lies in the identical action of $H$ and $H_{\text {free }}=-\partial_{\theta}^{2}$ on the periodic kernel.

$$
H e^{i m e^{i x} \sin \theta}=H_{\text {free }} e^{i m e^{i x} \sin \theta}=\left(m^{2} e^{2 i x}+\left(m \frac{d}{d m}\right)^{2}\right) e^{i m e^{i x} \sin \theta}
$$

While (57) is a well-known integral representation of the Bessel function 82], the interpretation as a canonical transformation is usually overlooked in most mathematical physics texts. That is unfortunate. This is an extremely straightforward and transparent connection between the quantum and classical theories that serves to distinguish this model. (It doesn't get any better than this! ${ }^{3}$ )

The integral transform is a two-to-one map from the space of free particle plane waves to Bessel functions $\left(e^{\mp i n \theta} \rightarrow( \pm 1)^{n} J_{n}\right.$ upon using $\left.J_{-n}=(-)^{n} J_{n}\right)$, just as happens in the real Liouville case $\left(e^{ \pm i k x} \rightarrow K_{i k}\right)$ [17. 15], although here we have selected normalizations for the wave functions so that the transformation uses the same normalization magnitude for all $n$ (with at most only an $n$-dependent phase), unlike the real Liouville integral tranformation, where the magnitude of the normalization varies with $k$ in order to obtain a conventionally normalized continuum wave function from the MacDonald function $K_{i k}$ (e.g. 26], 5th eqn.).

When restricted to the combinations $e^{i n \theta}+(-)^{n} e^{-i n \theta}$ the map is one-to-one and invertible. On the other hand, acting on $e^{i n \theta}-(-)^{n} e^{-i n \theta}$ the map projects to zero. When restricted either to the subspace of right-moving plane waves, or to the subspace of left-moving plane waves, the map is also one-to-one, but not invertible to the same subspace of plane waves. Inverting this transformation on $J_{n}$ by using the same kernel always gives a combination of left- and right-movers. (See the discussion and Table below.) This left-right combination is unavoidable, given the properties of the kernel $\exp \left(i m e^{i x} \sin \theta\right)$ and the symmetry $J_{-n}=(-)^{n} J_{n}$, although the combination of plane waves can be changed by choosing a different kernel in the integral representation of $J_{n}$. (See the discussion following the Table.). In any case, combinations of left- and right-movers result from inverting the map using the kernel we have chosen.

We note the effects of index raising/lowering operations on the complete integrand in (57).

$$
\left(-i \frac{\partial}{\partial x} \mp n\right) e^{i m e^{i x} \sin \theta-i n \theta}=\left(\mp i \frac{\partial}{\partial \theta} \mp m e^{i x} e^{\mp i \theta}\right) e^{i m e^{i x} \sin \theta-i n \theta}
$$

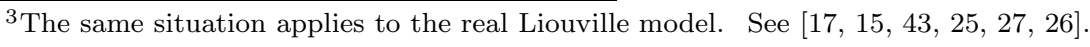


Thus, acting on the integral representation (57) and integrating away the $\theta$ derivative term, we obtain the usual recursion relations [82] only expressed for Bessel functions on the circle.

$$
\left(-i \frac{d}{d x} \mp n\right) J_{n}\left(m e^{i x}\right)=\mp m e^{i x} J_{n \pm 1}\left(m e^{i x}\right)
$$

We also note in passing that the exponentiated classical generating function has a well-known interpretation as an element of the Poincaré group on the plane (81] Chapter IV).

Inverting the transformations and the rôle of other classical trajectories in the quantized model The canonical integral transformation from Neumann polynomials to plane waves using the same kernel as in (57) warrants discussion. It is simply

$$
\frac{1}{2 \pi} \int_{0}^{2 \pi} e^{i m e^{i x} \sin \theta} A_{n}\left(m e^{i x}\right) d x= \begin{cases}1 & \text { for } n=0 \\ e^{i n \theta}+(-)^{n} e^{-i n \theta} & \text { for } n>0\end{cases}
$$

This follows from the biorthogonality of $\left\{J_{j}, A_{k}\right\}$, (52), and the well-known generating function (see [1] 9.1.41)

$$
e^{i z \sin \theta}=\sum_{n=-\infty}^{\infty} J_{n}(z) e^{i n \theta}=J_{0}(z)+\sum_{n=1}^{\infty} J_{n}(z)\left(e^{i n \theta}+(-1)^{n} e^{-i n \theta}\right)
$$

as well as $J_{-n}(z)=(-1)^{n} J_{n}(z)$. The transform (52) involves integration around the circle, $z=m e^{i x}$, $x \in[0,2 \pi]$, of a Neumann polynomial times the canonical transformation kernel. But, were we to make slight deformations of the circular contour (as allowed by the analytic form of the Neumann polynomials and the kernel in the neighborhood of the circle) the result of the transformation would be the same. Therefore, the transformation may be carried out just by integrating along a closed contour in the $z=m e^{i x}$ plane given exactly by following a single classical Liouville particle $x$-trajectory, for $E>0$.

To invert the transformation and go from plane waves to Neumann polynomials using the same kernel ultimately requires taking free particle plane waves off the circle and integrating up the imaginary axis. We may do this by first integrating along an arc of the circle to the point $\theta=-\alpha$, and then by following the path of a classical free particle trajectory for $\operatorname{Im} p_{\theta}>0, \operatorname{Re} p_{\theta}=0$ which evolves to $i \infty-\alpha$ as $t \rightarrow \infty$. Explicitly (82 $§ 9.14 \operatorname{Eqn}(3))$

$$
A_{n}(z)=\frac{1}{2} \varepsilon_{n} z \int_{0}^{\infty+i \alpha}\left(e^{n \vartheta}+(-1)^{n} e^{-n \vartheta}\right) e^{-z \sinh \vartheta} \cosh \vartheta d \vartheta
$$

where $|\alpha+\arg z|<\pi / 2$. Integrating by parts picks up a contribution at 0 when $n$ is even, but not when $n$ is odd, resulting in Schläfli's version of the Neumann polynomials (see §9.3-34 in [82]). Explicitly

$$
S_{n}(z) \equiv \frac{2}{n}\left(\frac{1}{\varepsilon_{n}} A_{n}(z)-\cos ^{2}(n \pi / 2)\right)=\int_{0}^{\infty+i \alpha}\left(e^{n \vartheta}-(-)^{n} e^{-n \vartheta}\right) e^{-z \sinh \vartheta} d \vartheta
$$

Alternatively, in terms of the original free particle variable $\theta=i \vartheta$.

$$
\begin{aligned}
S_{n}(z)= & \frac{2}{n}\left(\frac{1}{\varepsilon_{n}} A_{n}(z)-\cos ^{2}(n \pi / 2)\right)=i(-)^{n} \int_{0}^{i \infty-\alpha}\left(e^{i n \theta}-(-)^{n} e^{-i n \theta}\right) e^{i z \sin \theta} d \theta \\
A_{n}(z)= & \varepsilon_{n} \cos ^{2}(n \pi / 2)+i \varepsilon_{n}(-1)^{n} \frac{n}{2} \int_{0}^{i \infty-\alpha}\left(e^{i n \theta}-(-)^{n} e^{-i n \theta}\right) e^{i z \sin \theta} d \theta \\
& = \begin{cases}\varepsilon_{n}-n \varepsilon_{n} \int_{0}^{i \infty-\alpha} e^{i z \sin \theta} \sin (n \theta) d \theta & \text { for } n \text { even } \\
-i n \varepsilon_{n} \int_{0}^{i \infty-\alpha} e^{i z \sin \theta} \cos (n \theta) d \theta & \text { for } n \text { odd }\end{cases}
\end{aligned}
$$

At least three wedges (choices for $\alpha$ ) are required for an open cover of a full circle for $z$, although two wedges will cover all but two points on the $z$-circle.

We give a summary Table of the canonical integral transforms using the kernel $\exp \left(i m e^{i x} \sin \theta\right)$. The paths of integration are described in terms of relevant classical trajectories. In a well-defined sense as 
indicated in the Table, only integrations over particular, representative, classical trajectories are required to change wave functions for the free particle into those for the Liouville system, and vice versa.

\section{Integral Representations}

$J_{n}(z)=\frac{1}{4 \pi} \int_{0}^{2 \pi} e^{i z \sin \theta}\left(e^{-i n \theta}+(-1)^{n} e^{i n \theta}\right) d \theta$

$|\cos \theta| \int_{-\infty}^{\infty} e^{i z \sin \theta} J_{n}(z) d z=e^{i n \theta}+(-)^{n} e^{-i n \theta}$

$A_{n}(z)=\frac{\varepsilon_{n} z}{2 i} \int_{0}^{i \infty-\alpha} e^{i z \sin \theta}\left(e^{-i n \theta}+(-1)^{n} e^{i n \theta}\right) \cos \theta d \theta$

$\frac{1}{2 \pi i} \oint e^{i z \sin \theta} A_{n}(z) \frac{d z}{z}=\left(e^{i n \theta}+(-)^{n} e^{-i n \theta}\right) \frac{\varepsilon_{n}}{2}$ with

$z \equiv m e^{i x}$

$\theta$ real, $\sin \theta \neq \pm 1$,

$|\alpha+\arg z|<\pi / 2$

i.e. $|\alpha+x|<\pi / 2$

$\varepsilon_{n} \equiv 2-\delta_{n 0}$

\section{Relevant Trajectories}

$\theta$ on $\operatorname{Re} p_{\theta}>0, \operatorname{Im} p_{\theta}=0$ free particle trajectory

$x$ on $\operatorname{Re} E=0=\operatorname{Im} E$ $x(0)=0$ and $\pi$

Liouville trajectories

$\theta$ on $\operatorname{Re} p_{\theta}<0, \operatorname{Im} p_{\theta}=0, \theta(0)=0$ and $\operatorname{Re} p_{\theta}=0, \operatorname{Im} p_{\theta}>0, \theta(0)=\alpha$ free particle trajectories

$x$ on $\operatorname{Re} E>0, \operatorname{Im} E=0$

Liouville trajectory

Analyticity is in control here, in the Table. Up to some clearly discernible limitations, variations of the integration paths will not change the results. Indeed, some deformation of the integration path may be necessary to have it exactly coincide with the indicated classical trajectory (for example, in the last case).

As previously remarked, the first and last rows of the Table are immediate consequences of the biorthogonality of $\left\{J_{j}, A_{k}\right\}$, (52), the well-known generating function, (62), and $J_{-n}(z)=(-1)^{n} J_{n}(z)$ as well as $A_{-n}(z) \equiv(-1)^{n} A_{n}(z)$. The second row follows from the first row and the Fourier integral representation of the Dirac delta. This particular relation is sometimes written an as integral representation for the Chebyshev polynomials, after translating $\theta \rightarrow \theta+\pi / 2$ and defining $\omega \equiv \cos \theta$, whereupon it becomes (see 1 . 11.4.24)

$$
\cos (n \theta) \equiv T_{n}(\omega)=\frac{1}{2} i^{n} \sqrt{1-\omega^{2}} \int_{-\infty}^{\infty} e^{-i s \omega} J_{n}(s) d s \quad \text { for } \quad \omega^{2}<1
$$

The third row of the Table is obtained just by substituting $\theta=i \vartheta$ in (63), with no further insight on our part, but it may well have a more physical interpretation [46].

For the kernel being used, as presented in the Table, the map is $\left\{J_{n}\right\} \leftrightarrow\left\{e^{i n \theta}+(-)^{n} e^{-i n \theta}\right\} \leftrightarrow\left\{A_{n}\right\}$. The intermediate subspace of plane waves is not chiral. However, projections onto chiral subspaces may also be constructed.

Chiral kernels The result (62) is a bilinear but non-symmetric kernel that maps linear combinations of plane waves onto integer Bessel functions. Alternatively, the kernel maps Neumann polynomials onto equal mixtures of left- and right-moving plane waves. But obviously, we may project onto half the space of plane waves in an infinite number of other ways, say by choosing to keep in the sum over $n$ only one of $\exp ( \pm i n \theta)$, for all $n \geq 0$, and thereby produce other maps that act nontrivially on the complementary set of plane waves.

For example, the left-moving chiral subspace of plane waves is mapped one-to-one onto Bessel functions by the chiral kernel ${ }^{4}$

$$
S(z, \theta) \equiv \sum_{n=0}^{\infty} J_{n}(z) e^{i n \theta}
$$

Thus $S:\left\{e^{-i n \theta} \mid n \geq 0\right\} \rightarrow\left\{J_{n}(z)\right\}$. Alternatively, all Neumann polynomials are "right-mapped" by the

\footnotetext{
${ }^{4}$ The right-moving chiral subspace can be similarly mapped onto Bessels using $\widetilde{S}(z, \theta)=S(z,-\theta)$. Also note the radius of the free particle circle can be modified, $e^{i \theta} \rightarrow M e^{i \theta}$ as in $z=m e^{i x}$, by giving $\theta$ a constant imaginary part.
} 
kernel into the space of right-moving plane waves, $\left\{A_{n}(z)\right\}: S \rightarrow\left\{e^{i n \theta} \mid n \geq 0\right\}$. Explicitly

$$
J_{n}(z)=\frac{1}{2 \pi} \int_{0}^{2 \pi} S(z, \theta) e^{-i n \theta} d \theta, \quad e^{i n \theta}=\frac{1}{2 \pi i} \oint \frac{d z}{z} A_{n}(z) S(z, \theta)
$$

Moreover, note the null projective property on almost all of the right-moving subspace, $S:\left\{e^{i n \theta} \mid n>0\right\} \rightarrow$ $\{0\}$, as well as on all Bessel functions except for $J_{0},\left\{J_{n}(z) \mid n>0\right\}: S \rightarrow\{0\}$.

The chiral kernel can be expressed as special cases of Lommel's functions of two variables (82] $\$ 16.5-16.59$ )

$$
S(z, \theta)=U_{0}\left(i e^{i \theta} z, z\right)-i U_{1}\left(i e^{i \theta} z, z\right)
$$

but regrettably, there does not seem to be an elementary closed-form for $S$. From rewriting (62) as $\sum_{n=-\infty}^{\infty} t^{n} J_{n}(z)=\exp \frac{1}{2} z\left(t-\frac{1}{t}\right)$, however, an integral representation follows immediately.

$$
S(z, \theta)=\frac{1}{2 \pi i} \oint_{|t|>1} e^{\frac{1}{2} z\left(t-\frac{1}{t}\right)} \frac{1}{t-e^{i \theta}} d t
$$

Here the $t$ contour is counterclockwise around the origin with $|t|>1 .^{5}$ The simple appearance of (170) is somewhat deceptive.

As in the case of the previous kernel, $e^{i z \sin \theta}$, this $S$ establishes a quantum equivalence between complex Liouville and free particle systems. Only now the equivalence is with free chiral particles on a circle. Once again, this is directly evident from the identical action of $H$ and $H_{\text {free }}=p_{\theta}^{2}$ on the kernel.

$$
H S(z, \theta)=H_{\text {free }} S(z, \theta)=\sum_{n=0}^{\infty} n^{2} J_{n}(z) e^{i n \theta}
$$

This may be checked against the partial differential equations satisfied by the Lommel functions in (69), or the contour representation in (70). From either of those results, or by manipulating the sum in (71), there follow other forms for this bilocal Hamiltonian kernel acting on the two spaces.

The chiral kernel can be inverted on the space of Bessel functions by the formal kernel $S^{-1}(\theta, z)$.

$$
S^{-1}(\theta, z) \equiv \sum_{n=0}^{\infty} e^{-i n \theta} A_{n}(z), \quad e^{-i n \theta}=\frac{1}{2 \pi i} \oint \frac{d z}{z} S^{-1}(\theta, z) J_{n}(z)
$$

Thus $S^{-1}:\left\{J_{n}(z)\right\} \rightarrow\left\{e^{-i n \theta} \mid n \geq 0\right\}$, and therefore $S^{-1} S=1:\left\{e^{-i n \theta} \mid n \geq 0\right\} \rightarrow\left\{e^{-i n \theta} \mid n \geq 0\right\}$ and $S S^{-1}=1:\left\{J_{n}(z)\right\} \rightarrow\left\{J_{n}(z)\right\}$. Alternatively, $\left\{e^{i n \theta} \mid n \geq 0\right\}: S^{-1} \rightarrow\left\{A_{n}(z)\right\}$ since formally

$$
A_{n}(z)=\frac{1}{2 \pi} \int_{0}^{2 \pi} e^{i n \theta} S^{-1}(\theta, z) d \theta
$$

The Liouville and free Hamiltonians act differently on $S^{-1}(\theta, z)$ due to the inhomogeneous terms in (54). Acting term-by-term and summing the formal series we find

$$
\begin{aligned}
\left(H-H_{\text {free }}\right) S^{-1}(\theta, z) & =2 z i \partial_{\theta} \sum_{\text {odd } n>0} e^{-i n \theta}+2 z^{2} \sum_{\text {even } n \geq 0} e^{-i n \theta} \\
& =\frac{2 z e^{-i \theta}\left(1+e^{-2 i \theta}\right)+2 z^{2}\left(1-e^{-2 i \theta}\right)}{\left(1-e^{-2 i \theta}\right)^{2}}
\end{aligned}
$$

This has singularities as a function of $\theta$, but these can be avoided in any integrations by changing the radius of the free particle circle (i.e. by taking $\operatorname{Im} \theta \neq 0$.) The RHS of (744) is also manifestly orthogonal to the right sector upon integration $\oint \frac{d z}{z}$.

This formal inverse allows us to express the equivalence to a free chiral particle in a somewhat heuristic, but very concise way.

$$
H_{\text {free chiral }}=S^{-1} H S
$$

\footnotetext{
${ }^{5}$ If $|t|<1$ for the contour, instead of $S$ it yields $1-\widetilde{S}(z, \theta+\pi)$.
} 
Or more explicitly

$$
H_{\text {free chiral }}\left(\theta^{\prime}, \theta\right)=\frac{1}{2 \pi i} \oint \frac{d z}{z} S^{-1}\left(\theta^{\prime}, z\right) H S(z, \theta)=\sum_{n=0}^{\infty} e^{-i n \theta^{\prime}} n^{2} e^{i n \theta}=\frac{e^{i\left(\theta-\theta^{\prime}\right)}\left(1+e^{i\left(\theta-\theta^{\prime}\right)}\right)}{\left(1-e^{i\left(\theta-\theta^{\prime}\right)}\right)^{3}}
$$

The last expression here is the usual form for the free chiral Hamiltonian kernel, and it is manifestly not local, whereas the complex $H$ is local.

As a technical point, we note that $S^{-1}(\theta, z)$ is indeed a formal kernel, since it is a divergent series, given the normalizations of the Neumann polynomials. Nevertheless, it can be Borel summed in closed form (a result due to Kapteyn, see [82], §9.16).

$$
\sum_{n=0}^{\infty} t^{n} A_{n}(z)=\left(1+t^{2}\right) z \int_{0}^{\infty} \frac{e^{-u} d u}{\left(1-t^{2}\right) z-2 t u}
$$

where the integral is recognizable as the exponential integral function Ei. Conversely, the sum in (77) is the asymptotic expansion of the integral, the latter being well-defined and obviously convergent when $\operatorname{Re}\left(1-t^{2}\right) z / t<0$. As generally remarked following (13), the convergence of this generating function for the dual polynomials can be improved by making compensating changes in the normalizations: $\left\{J_{j}, A_{k}\right\} \rightarrow$ $\left\{Z_{j} J_{j}, A_{k} / Z_{k}\right\}$. Thus $Z_{n}$ can be chosen to make $\sum_{n=0}^{\infty} \frac{1}{t^{n}} A_{n}(z) / Z_{n}$ absolutely, uniformly, and rapidly convergent. The price to be paid is that $\sum_{n=0}^{\infty} Z_{n} J_{n}(z) e^{i n \theta}$ will then be a divergent series. ${ }^{6}$

Other non-symmetric kernels may also be constructed. For simple examples, reconsider those in (23) and (25). Of greater interest, perhaps, are symmetric kernels given as sums of bilinears where both functions are of the same type. It is often possible to express these in closed form as well.

A simple kernel for the bilocal norm While it may be difficult to find a closed form for the kernel $K(x, y)$, since our normalizations for the $A_{n}\left(m e^{i x}\right)$ make this a divergent series, it is a simple task to find a closed form for $J(x, y)$, the inverse of $K$ on the spaces of interest. We recall Neumann's addition theorem, (27) and (28), only this time we choose more convenient normalizations to write

$$
J(x, y) \equiv J_{0}\left(m e^{-i x}-m e^{i y}\right)=\sum_{n=0}^{\infty} \varepsilon_{n} J_{n}\left(m e^{-i x}\right) J_{n}\left(m e^{i y}\right)
$$

where $\varepsilon_{0}=1, \varepsilon_{n \geq 1}=2$. Formally, the determinant of this kernel is

$$
\begin{aligned}
\operatorname{det}(J) & =\exp (\operatorname{Tr} \ln J)=\exp \int_{0}^{2 \pi} \ln (J(x, x)) d x \\
& =\exp \int_{0}^{2 \pi} \ln \left(J_{0}(2 i m \sin x)\right) d x \\
& =\exp \int_{0}^{2 \pi} \ln \left(I_{0}(2 m \sin x)\right) d x
\end{aligned}
$$

where $I_{0}$ is the modified Bessel function (see (93) below). Thus det $(J)$ never vanishes, for any real $m$, implying the existence of the formal inverse for real $m$ and $x \in[0,2 \pi]$. With the normalizations in (78) that

${ }^{6}$ The most "balanced" asymptotic index behavior for rescaled eigenfunctions and their duals is achieved as follows.

$$
n ! 2^{n} J_{n}(z) \underset{n \rightarrow \infty}{\simeq} z^{n}, \quad \frac{1}{n ! 2^{n}} A_{n}(t) \underset{n \rightarrow \infty}{\sim} \frac{1}{t^{n}}
$$

Then the leading behavior of the terms in the bilinear sums, modified accordingly, is

$$
\begin{gathered}
\frac{1}{(w z)^{N}} \sum_{n=N}^{\infty}\left(n ! 2^{n} J_{n}(w)\right)\left(n ! 2^{n} J_{n}(z)\right) \underset{N \rightarrow \infty}{\widetilde{1}} \frac{1}{(w z)^{N}} \sum_{n=N}^{\infty} w^{n} z^{n}=\frac{1}{1-w z} \\
(s t)^{N} \sum_{n=N}^{\infty}\left(\frac{1}{n ! 2^{n}} A_{n}(s)\right)\left(\frac{1}{n ! 2^{n}} A_{n}(t)\right) \underset{N \rightarrow \infty}{\widetilde{N}}(s t)^{N} \sum_{n=N}^{\infty} \frac{1}{s^{n} t^{n}}=\frac{1}{1-\frac{1}{s t}}
\end{gathered}
$$


formal inverse is

$$
K(x, y)=\sum_{n=0}^{\infty} \frac{1}{\varepsilon_{n}} A_{n}\left(m e^{i x}\right) A_{n}\left(m e^{-i y}\right)
$$

These exact results should be compared to those for the spectral resolvent, in models where $V(x)=(i x)^{N}$ [54, 7, 35, 77].

As in (13), the manifestly hermitian kernel $J(x, y)$ can be used to evaluate the norms of arbitrary $2 \pi$ periodic states

$$
\psi(x) \equiv \sum_{n=0}^{\infty} c_{n} \sqrt{\varepsilon_{n}} J_{n}\left(m e^{i x}\right)
$$

through use of the corresponding dual functions

$$
\begin{gathered}
\psi_{\text {dual }}(x) \equiv \sum_{n=0}^{\infty} c_{n}^{*} A_{n}\left(m e^{i x}\right) / \sqrt{\varepsilon_{n}} \\
\|\psi\|^{2}=\frac{1}{(2 \pi)^{2}} \int_{0}^{2 \pi} d x \int_{0}^{2 \pi} d y \overline{\psi_{\text {dual }}(x)} J(x, y) \quad \psi_{\text {dual }}(y)=\sum_{n=0}^{\infty}\left|c_{n}\right|^{2}
\end{gathered}
$$

We note that $J(x, y)$ converts the dual polynomials into conjugated Bessel functions, and conjugated polynomials into Bessels. ${ }^{7}$

$$
\begin{aligned}
& J_{n}^{*}\left(m e^{i x}\right)=J_{n}\left(m e^{-i x}\right)=\frac{1}{2 \pi \varepsilon_{n}} \int_{0}^{2 \pi} d y J(x, y) A_{n}\left(m e^{i y}\right) \\
& J_{n}\left(m e^{i y}\right)=\frac{1}{2 \pi \varepsilon_{n}} \int_{0}^{2 \pi} d x A_{n}\left(m e^{-i x}\right) J(x, y)
\end{aligned}
$$

Hamiltonian kernel on the dual space We also note that $J(x, y)$ converts $H$ into $H^{*}$ to produce a bilocal Hamiltonian kernel equivalent to either, in the following sense.

$$
\left(-\frac{\partial^{2}}{\partial x^{2}}+m^{2} e^{-2 i x}\right) J(x, y)=\left(-\frac{\partial^{2}}{\partial y^{2}}+m^{2} e^{2 i y}\right) J(x, y)=\sum_{n=0}^{\infty} n^{2} \varepsilon_{n} J_{n}\left(m e^{-i x}\right) J_{n}\left(m e^{i y}\right)
$$

The sum converges absolutely, uniformly, and very rapidly. Evaluating this sum in closed form produces an explicit hermitian kernel.

$$
H(x, y)=H^{*}(y, x)=m \frac{J_{1}\left(m e^{-i x}-m e^{i y}\right)}{e^{-i y}-e^{i x}}=\sum_{n=0}^{\infty} n^{2} \varepsilon_{n} J_{n}\left(m e^{-i x}\right) J_{n}\left(m e^{i y}\right)
$$

As a series in $m\left(e^{-i x}-e^{i y}\right)$ this bilocal Hamiltonian is rapidly convergent

$$
H(x, y)=\frac{m^{2}}{2} e^{-i(x-y)} \sum_{n=0}^{\infty} \frac{\left(-m^{2}\right)^{n}\left(e^{-i x}-e^{i y}\right)^{2 n}}{4^{n} n !(n+1) !}
$$

and as a series in $e^{-i x}$ or $e^{i y}$, it only involves non-negative powers of either. This Hamiltonian kernel acts on the dual space to give energy operator matrix elements, or averages as in (15).

$$
\langle H\rangle_{\psi}=\frac{1}{(2 \pi)^{2}\|\psi\|^{2}} \int_{0}^{2 \pi} d x \int_{0}^{2 \pi} d y \overline{\psi_{\text {dual }}(x)} H(x, y) \quad \psi_{\text {dual }}(y)=\sum_{n=0}^{\infty} n^{2}\left|c_{n}\right|^{2} /\|\psi\|^{2}
$$

It is sometimes convenient to re-express this in terms of the action of $H(x, y)$ on the dual polynomials, similar to (84).

$$
n^{2} J_{n}^{*}\left(m e^{i x}\right)=n^{2} J_{n}\left(m e^{-i x}\right)=\frac{1}{2 \pi \varepsilon_{n}} \int_{0}^{2 \pi} d y H(x, y) A_{n}\left(m e^{i y}\right)
$$

\footnotetext{
${ }^{7}$ Simply changing the sign of $x$ in $J(x, y)$ yields a non-hermitian kernel that converts $A_{n}\left(m e^{i y}\right)$ into non-conjugated $J_{n}\left(m e^{i x}\right)$.
} 
Another map between $H$ and $H^{*}$ on $2 \pi$-periodic functions Consider [26] integral kernels depending on a parameter $s$. In particular, consider the very simple kernel

$$
\begin{aligned}
K(x, y ; s) & \equiv \exp k(x, y ; s) \\
k(x, y ; s) & \equiv \frac{m}{2}\left(\frac{1}{s} e^{i(-x+y)}-s e^{i(-x-y)}-s e^{i(x+y)}\right) \\
k(x, y ; s) & =k^{*}(y, x ; s) \quad \text { for } s \in \mathbb{R}
\end{aligned}
$$

All terms in these expressions are periodic in $x$ and $y$, separately, with period $2 \pi$. Once again we have

$$
\left(-\frac{\partial^{2}}{\partial x^{2}}+m^{2} e^{-2 i x}\right) K(x, y ; s)=\left(-\frac{\partial^{2}}{\partial y^{2}}+m^{2} e^{2 i y}\right) K(x, y ; s)
$$

So this kernel also accomplishes the task of converting $H$ into $H^{*}$ and vice versa. Using this kernel, we find the integral transforms ${ }^{8}$

$$
\begin{aligned}
& J_{n}^{*}\left(m e^{i x}\right)=J_{n}\left(m e^{-i x}\right)=\frac{1}{2 \pi} \frac{(-1)^{n}}{I_{n}(m s)} \int_{0}^{2 \pi} K(x, y ; s) J_{n}\left(m e^{i y}\right) d y \\
& J_{n}\left(m e^{i y}\right)=\frac{1}{2 \pi} \frac{(-1)^{n}}{I_{n}(m s)} \int_{0}^{2 \pi} J_{n}\left(m e^{-i x}\right) K(x, y ; s) d x
\end{aligned}
$$

which should be compared to (84). For real $m s \neq 0$, the modified Bessel $I_{n}(m s)$ is real and it has no zeroes, as is evident in

$$
I_{\mu}(m s)=\left(\frac{m s}{2}\right)^{\mu} \sum_{k=0}^{\infty} \frac{1}{k ! \Gamma(1+\mu+k)}\left(\frac{m s}{2}\right)^{2 k}
$$

For all real $m$ and $s, K(x, y ; s)$ is an hermitian kernel, $K(x, y ; s)=K(y, x ; s)^{*}$, and it provides an explicit, one-to-one, invertible, closed-form transformation between the eigenfunctions of $H$ and $H^{*}$.

Another way to state the result (92) is as an expansion of the kernel in terms of Bessel/Neumann trilinears. (A similar relation holds for real Liouville theory. See [26].) From the transforms given, and the completeness of the $J_{n}(z)$ on positive powers of $z$, we deduce that

$$
\begin{aligned}
K(x, y ; s) & =J_{0}\left(m e^{-i x}\right) I_{0}(m s) J_{0}\left(m e^{i y}\right) \\
& +\sum_{n=1}^{\infty}(-1)^{n} J_{n}\left(m e^{-i x}\right) I_{n}(m s) A_{n}\left(m e^{i y}\right)+(-1)^{n} A_{n}\left(m e^{-i x}\right) I_{n}(m s) J_{n}\left(m e^{i y}\right) \\
& +\sum_{j, k=1}^{\infty} J_{j}\left(m e^{-i x}\right) c_{j k}(s) J_{k}\left(m e^{i y}\right)
\end{aligned}
$$

where we have not determined the coefficients $c_{j k}(s)$ (except for the omitted $c_{00}=0=c_{01}=c_{10}$ ).

More information may be incorporated into the sums expressing $K(x, y ; s)$ by imposing total symmetry among $e^{-i x}, e^{i y}$, and $i s \equiv e^{i z}$. Since $J_{n}\left(m e^{i z}=i m s\right)=i^{n} I_{n}\left(m s=-i m e^{i z}\right)$ we have

$$
\begin{gathered}
J_{n}\left(m e^{i x}\right) J_{n}\left(m e^{i y}\right)=\frac{1}{2 \pi i^{n}} \int_{0}^{2 \pi} S(x, y, z) J_{n}\left(m e^{i z}\right) d z \\
S(x, y, z) \equiv \exp \left[\frac{i m}{2}\left(e^{i(x+y-z)}+e^{i(x-y+z)}+e^{i(-x+y+z)}\right)\right]=K\left(-x, y ; s=-i e^{i z}\right)
\end{gathered}
$$

where $S$ is unchanged by permutations of its arguments. It follows as above that this symmetric kernel is

\footnotetext{
${ }^{8}$ A similar kernel may be used in real Liouville theory to effect an integral transformation from one $K_{\nu}$ to another, leading to MacDonald's identity (82 §13.71). See [26] and references therein. We were not able to find 92$]$ in the literature. But it is not too difficult to work it out.
} 
of the form

$$
\begin{gathered}
S(x, y, z)=J_{0}\left(m e^{i x}\right) J_{0}\left(m e^{i y}\right) J_{0}\left(m e^{i z}\right)+\sum_{j, k, n=1}^{\infty} c_{j k n} J_{j}\left(m e^{i x}\right) J_{k}\left(m e^{i y}\right) J_{n}\left(m e^{i z}\right) \\
+\sum_{n=1}^{\infty} i^{n} A_{n}\left(m e^{i x}\right) J_{n}\left(m e^{i y}\right) J_{n}\left(m e^{i z}\right)+i^{n} J_{n}\left(m e^{i x}\right) A_{n}\left(m e^{i y}\right) J_{n}\left(m e^{i z}\right)+i^{n} J_{n}\left(m e^{i x}\right) J_{n}\left(m e^{i y}\right) A_{n}\left(m e^{i z}\right)
\end{gathered}
$$

We have not determined the symmetric coefficients $c_{j k n}$. Regardless of their values, they drop out of (95a). Their relation to the previous coefficients is

$$
c_{j k}(s)=\delta_{j k} i^{k} A_{k}(i m s)+\sum_{n=1}^{\infty} c_{j k n} i^{n} I_{n}(m s)
$$

The $c_{j k n}$ could be determined by evaluating $\int_{0}^{2 \pi} S(x, y, z) A_{n}\left(m e^{i z}\right) d z$ through a direct calculation.

Classical behavior of time-dependent expectation values As a final comment on the structure of this model, we remark that it is straightforward to take linear combinations of energy eigenstates to produce near-classical time-dependent behavior for expectation values, including those which may be complex, such as

$$
\begin{aligned}
\langle p\rangle_{\psi}(t) & \equiv \frac{1}{2 \pi\|\psi\|^{2}} \int_{0}^{2 \pi} d y \psi_{\text {dual }}(y ; t)\left(-i \partial_{y} \psi(y ; t)\right) \\
& =\frac{1}{(2 \pi)^{2}\|\psi\|^{2}} \int_{0}^{2 \pi} d x \int_{0}^{2 \pi} d y \overline{\psi_{\text {dual }}(x ; t)}\left(-i \partial_{y} J(x, y)\right) \psi_{\text {dual }}(y ; t)
\end{aligned}
$$

Here we have defined the time-dependent function and its dual as expected from (81) and (82).

$$
\psi(x ; t)=\sum_{n=0}^{\infty} c_{n} e^{-i n^{2} t} \sqrt{\varepsilon_{n}} J_{n}\left(m e^{i x}\right), \quad \psi_{\text {dual }}(x ; t)=\sum_{n=0}^{\infty} c_{n}^{*} e^{i n^{2} t} A_{n}\left(m e^{i x}\right) / \sqrt{\varepsilon_{n}}
$$

We have also used $\psi(y ; t)=\frac{1}{2 \pi} \int_{0}^{2 \pi} d x \overline{\psi_{\text {dual }}(x ; t)} J(x, y)$ to write the expectation value in terms of the non-hermitian bilocal kernel $-i \partial_{y} J(x, y)$.

In fact, a simple combination of only two energy eigenstates will give $\langle p\rangle_{\psi}(t)$ that evolves around a circle in the complex plane, whose center is on the real axis, with constant angular velocity around the circle. We believe that only slightly more complicated coherent states [52] can be constructed to reproduce the exact time-dependence evident in the classical motions, where the angular velocity around the momentum circle is not constant. All this is in accord with the time derivative

$$
\begin{aligned}
\frac{d}{d t}\langle p\rangle_{\psi}(t) & =-i\langle[p, H]\rangle_{\psi}(t) \equiv \frac{-i}{2 \pi\|\psi\|^{2}} \int_{0}^{2 \pi} d x \psi_{\text {dual }}(x ; t)[p, H] \psi(x ; t) \\
& =-2 i m^{2}\left\langle e^{2 i x}\right\rangle_{\psi}(t)
\end{aligned}
$$

Classical evolution of $\langle p\rangle_{\psi}(t)$ follows if the state $\psi$ is such that the last RHS evolves classically. Since we also have

$$
\frac{d}{d t}\left\langle e^{2 i x}\right\rangle_{\psi}(t)=-i\left\langle\left[e^{2 i x}, H\right]\right\rangle_{\psi}(t)=2 i\left\langle\left\{p, e^{2 i x}\right\}\right\rangle_{\psi}(t)
$$

classical behavior is guaranteed to the extent that $\left\langle\left\{p, e^{2 i x}\right\}\right\rangle_{\psi}(t)$ coincides with $2\langle p\rangle_{\psi}(t)\left\langle e^{2 i x}\right\rangle_{\psi}(t)$. (This is just Ehrenfest's theorem for this biorthogonal system.) In this regard, it is crucial that we are able to replace $n^{2} A_{n}$ by $H A_{n}$ under the integrations over $x$ in (101) and (102). Fortunately, the inhomogeneous terms in (54) do not contribute to the expectations under consideration, so the replacement is permitted among the integrands in these cases.

Remnants of classical behavior or the classical limits of quantum systems are often most clearly understood from the point of view of phase-space quantum mechanics, i.e. deformation quantization [86]. In that 
framework, let us briefly return to the issue of the anisotropic brackets. The classical anisotropic brackets described earlier are just $\hbar \rightarrow 0$ limits of Moyal brackets built from anisotropic Groenewold products acting on phase-space distributions, where the phase-space variables are allowed to be complex. Thus

$$
[f(x, p), g(x, p)]_{\mathrm{AB}}=\lim _{\hbar \rightarrow 0} \frac{1}{i \hbar}(f * g-g * f)
$$

where the associative but non-commutative anisotropic product is given in its most symmetrical form by

$$
* e^{i \frac{\hbar}{4}\left(\overleftarrow{\partial}_{\operatorname{Re} x} \vec{\partial}_{\operatorname{Re} p}-\overleftarrow{\partial}_{\operatorname{Re} p} \vec{\partial}_{\operatorname{Re} x}\right)-i \frac{\hbar}{4}\left(\overleftarrow{\partial}_{\operatorname{Im} x} \vec{\partial}_{\operatorname{Im} p}-\overleftarrow{\partial}_{\operatorname{Im} p} \vec{\partial}_{\operatorname{Im} x}\right)}
$$

Clearly then, $x * p=x p+i \hbar / 2, p * x=x p-i \hbar / 2$, and $[x, p]_{\mathrm{AB}}=1$ as in (41), as well as $[\operatorname{Re} x, \operatorname{Re} p]_{\mathrm{AB}}=1 / 2$, $[\operatorname{Im} x, \operatorname{Im} p]_{\mathrm{AB}}=-1 / 2$, etc. Therefore the previous continuation of the classical dynamics is recovered in the $\hbar \rightarrow 0$ limit. In fact, the quantization of the system can be carried out in full detail on the complex phase-space through the use of this star product acting on Wigner functions built from bilinears in the wave functions and their duals. (A thorough discussion of this approach to quantization is in preparation [28].)

\section{$5 \quad$ Magnetic field effects}

Magnetic fields can produce some remarkable changes in the structure of a complex biorthogonal system, even in the simple physical set-up where charged particles move around a solenoid containing magnetic flux $\Phi$ with only a constant vector potential $A \propto \Phi$ on the surface of the solenoid, in addition to the complex potential energy imagined to be already present. ${ }^{9}$ As an example, we add such magnetic effects minimally to the previous Hamiltonian, to obtain the form $(\nu \equiv-e A / c)$

$$
H=(p+\nu)^{2}+m^{2} e^{2 i x}
$$

Here $p$ is the momentum canonically conjugate to $x$, so classically there is no discernible change from the case $\nu=0$. Classically, we have $d x / d t=[x, H]=2(p+\nu)$, so $H=\frac{1}{4}(d x / d t)^{2}+m^{2} e^{2 i x}$, and for a given energy the trajectories are the same as obtained in the $\nu=0$ case. A corresponding classical canonical transformation taking $H \rightarrow H_{\text {free }}=p_{\theta}^{2}$ is now given by

$$
F=-\nu x+m e^{i x} \sin \theta, \quad p \equiv \frac{\partial}{\partial x} F=-\nu+i m e^{i x} \sin \theta, \quad p_{\theta} \equiv-\frac{\partial}{\partial \theta} F=-m e^{i x} \cos \theta
$$

Thus there are no significant changes, classically.

But consider the QM of this model, and consider only $2 \pi$-periodic solutions, since the effects of $\nu \neq 0$ are most dramatic in this situation. As an operator

$$
H=\left(-i \partial_{x}+\nu\right)^{2}+m^{2} e^{2 i x}
$$

and for generic $\nu$ the set of periodic eigenfunctions now consists of two sectors given by

$$
H e^{-i \nu x} J_{\nu \pm n}\left(m e^{i x}\right)=(\nu \pm n)^{2} e^{-i \nu x} J_{\nu \pm n}\left(m e^{i x}\right)
$$

for $n \in \mathbb{Z}_{\geq 0}$. These eigenfunctions are all even under PT, for real $\nu$. For want of better terms, we will call these the right and left sectors since for generic $\nu$ all right-moving and left-moving plane waves are contained in the spans of $\left\{e^{-i \nu x} J_{\nu+n} \mid n \in \mathbb{Z}_{\geq 0}\right\}$ and $\left\{e^{-i \nu x} J_{\nu-n} \mid n \in \mathbb{Z}_{\geq 0}\right\}$, respectively. ${ }^{10}$ When $\nu \notin \mathbb{Z}, J_{\nu \pm n}$ give two independent states for all $n>0$. Thus the number of states has doubled from the $\nu=0$ situation, no matter how small $\nu \neq 0$ is. ${ }^{11}$ The effect of $\nu \notin \mathbb{Z}$ is to restore all left-moving plane waves to the span of the energy eigenfunctions, in sharp contrast to $\nu=0$ where only the right-moving plane waves could be expanded in $J_{n}\left(m e^{i x}\right)$.

\footnotetext{
${ }^{9}$ Alternatively, non-hermitian $H$ could be obtained just by considering complex vector potentials for a particle on the plane, without any exponential interactions. See 39].

${ }^{10}$ Yes, $n=0$ is in both sectors, as defined. If this is troubling, then feel free to define three sectors to avoid any overlap.

${ }^{11}$ This $2 \rightarrow 1$ accounting of the states, as $\nu \rightarrow 0$, is complementary to the $1 \rightarrow 2$ state counting for linear "wall" potentials, as the slope of the walls goes to zero.
} 
So the energies are given on the two sectors by

$$
E_{ \pm n}=(\nu \pm n)^{2} \quad \text { for } \quad n=0,1, \cdots
$$

For generic $\nu, E_{ \pm n}$ are not degenerate. But if $\nu-1 / 2=\lambda \in \mathbb{Z}$, eigenvalues are doubly degenerate. For example, if $\nu=1 / 2$, the degenerate pairs of energies are $E_{0,-1}, E_{1,-2}$, etc. In this case there are two independent states for each energy.

Otherwise, when $\nu \in \mathbb{Z}$, most if not all of the $J_{\nu-n}$ states meld into the $J_{\nu+n}$ states $^{12}$, since the Bessels with negative integer index are not independent of those with positive integer index, i.e. $J_{\nu-n}(z)=$ $(-1)^{\nu-n} J_{n-\nu}(z)$, for $n$ and $\nu \in \mathbb{Z}$. Sorting out the independent functions leaves just $\left\{J_{\lambda} \mid \lambda \in \mathbb{Z}_{\geq-\nu}\right\}$. For instance, when $\nu \in \mathbb{Z}_{\geq 0}$ the independent states are $e^{-i \nu x} J_{\nu-n}$ for $\nu-n \in\{0,1, \cdots, \nu\}$ (alternatively $n \in\{\nu, \nu-1, \cdots, 1,0\})$ and $e^{-i \nu x} J_{\nu+n}$ for $n \in \mathbb{Z}_{\geq 1}$. That is to say, when $\nu \in \mathbb{Z}_{\geq 0}$, the independent states are nondegenerate with energies $0,1,4,9, \cdots$, exactly as in the $\nu=0$ situation (although the wave functions are different for $\nu=0$ and $\nu \neq 0$ ). Moreover, when $\nu \in \mathbb{Z}_{\geq 0}$ only a finite number of left-moving plane waves are in the span of the energy eigenfunctions, namely: $\left\{e^{-i \nu x}, e^{-i(\nu-1) x}, \cdots, e^{-i x}\right\}$. Similar results apply when $\nu \in \mathbb{Z}_{<0}$, although in this case only right-moving plane waves are spanned by the eigenfunctions, namely: $\left\{e^{i|\nu| x}, e^{i(1+|\nu|) x}, e^{i(2+|\nu|) x} \cdots\right\}$, with the first $|\nu|$ right-movers absent from the span. But again, the energies are $0,1,4,9, \cdots$, exactly as in the $\nu=0$ situation. In any case, all of these situations involving integer $\nu$ are (gauge) equivalent through energy preserving one-to-one maps connecting the various Bessel functions. Map details are left to the reader to establish.

For generic $\nu$, the dual space may be constructed for all eigenfunctions, but again not by the naive PT method. Every function in the right sector, $e^{-i \nu x} J_{\nu+n}\left(m e^{i x}\right)$, is once again self-orthogonal (except $n=0)$ as is every function in the left sector. So, an appropriate dual function is not proportional to the eigenfunction, rather it is given by $e^{i \nu x} J_{-\nu-n}\left(m e^{i x}\right) / C_{n}$ where $C_{n}=\frac{1}{2 \pi} \int_{0}^{2 \pi} J_{-\nu-n}\left(m e^{i x}\right) J_{\nu+n}\left(m e^{i x}\right) d x$. This dual function is not an eigenfunction of $H$. Instead, it is an eigenfunction of

$$
\widetilde{H}=\left(-i \partial_{x}-\nu\right)^{2}+m^{2} e^{2 i x}
$$

obtained by flipping the sign of the vector potential ${ }^{13}$, with the same eigenvalue as the associated right-sector function,

$$
\widetilde{H} e^{i \nu x} J_{-\nu-n}\left(m e^{i x}\right)=(\nu+n)^{2} e^{i \nu x} J_{-\nu-n}\left(m e^{i x}\right)
$$

Similarly the dual for every left-sector eigenfunction, say $e^{-i \nu x} J_{\nu-n}\left(m e^{i x}\right)$, is $e^{i \nu x} J_{-\nu+n}\left(m e^{i x}\right) / D_{n}$ where $D_{n}=\frac{1}{2 \pi} \int_{0}^{2 \pi} J_{-\nu+n}\left(m e^{i x}\right) J_{\nu-n}\left(m e^{i x}\right) d x$, and $\widetilde{H} e^{i \nu x} J_{-\nu+n}\left(m e^{i x}\right)=(\nu-n)^{2} e^{i \nu x} J_{-\nu+n}\left(m e^{i x}\right)$. The duals for right- and left-sector eigenfunctions are guaranteed to be orthogonal to left- and right-sector eigenfunctions, respectively, for generic $\nu$ where $E_{ \pm n}$ are not degenerate, by standard arguments (as in (54) et seq.). But if $\nu-1 / 2=\lambda \in \mathbb{Z}$, further consideration is needed, since right- and left-sector eigenvalues are degenerate. An adaptation to biorthogonal systems of the usual degenerate state methods in conventional QM suffices to handle this situation for both right and left sectors, in a straightforward way.

Canonical integral representations for the wave functions and their duals may also be exhibited for this model, as given for example by (47) with $\sqrt{E}$ replaced by $\nu \pm n$ and $-\nu \pm n$.

Rather than dwell on this, for simplicity we now limit our discussion to the right sector for any $\nu \notin \mathbb{Z}_{<0}$. This is an invariant subspace spanned by $\left\{e^{-i \nu x} J_{\nu+n}\left(m e^{i x}\right) \mid n=0,1, \cdots\right\}$, and as previously pointed out, all right-moving plane waves are contained in this subspace for $\nu \notin \mathbb{Z}_{<0}$. As an alternative to calling it the right sector, it is the analytic sector in the variable $z=m e^{i x}$. For this subspace, rather than as eigenfunctions of $\widetilde{H}$, the duals may be constructed as finite polynomials in $1 / z$ in a manner that closely parallels our previous analysis of the $\nu=0$ situation for $2 \pi$-periodic functions.

Gegenbauer's generalization of the Neumann polynomials and the corresponding construction of the dual space In this generalization, the index $n$ is always $\geq 0$ and integral, while the index $\nu$ is

\footnotetext{
${ }^{12}$ That is to say, integer $\nu$ are exceptional spectral points. See [50 47.

${ }^{13}$ Note this is not what would happen under CPT, where "C" here really is charge conjugation, since $\nu \propto e A$ and this does not change sign under CPT.
} 
allowed any value except $-1,-2,-3, \cdots$. Negative $\nu$ are allowed, just so long as they are not integral. An explicit definition is $(82] \S 9.2)$

$$
\begin{aligned}
A_{n, \nu}(w) & =\frac{2^{\nu+n}(\nu+n)}{w^{n+1}} \sum_{k=0}^{\lfloor n / 2\rfloor} \frac{\Gamma(\nu+n-k)}{k !}\left(\frac{w}{2}\right)^{2 k} \\
& =\frac{2^{\nu+n} \Gamma(\nu+n+1)}{w^{n+1}}\left(1+\frac{w^{2} / 4}{(\nu+n-1)}+\frac{w^{4} / 16}{2 !(\nu+n-1)(\nu+n-2)}+\cdots\right)
\end{aligned}
$$

The Neumann polynomials are a special case: $A_{n, \nu=0}(w)=\varepsilon_{n} O_{n}(w) . \quad\left(\mathrm{NB} A_{n, \nu=0}(w)=\frac{1}{w} A_{n}(w)\right.$ in our previous notation. Sorry about that!) The Fourier transform on a simple counter-clockwise contour enclosing the origin once is

$$
\frac{1}{2 \pi i} \oint e^{i z w} A_{n, \nu}(w) d w=2^{\nu} i^{n}(\nu+n) \Gamma(\nu) C_{n}^{\nu}(z) \quad \text { where } \quad \frac{1}{\left(1-2 t z+t^{2}\right)^{\nu}} \equiv \sum_{n=0}^{\infty} t^{n} C_{n}^{\nu}(z)
$$

That is to say, the contour Fourier transforms of Gegenbauer's polynomials $A_{n, \nu}$ are just the Gegenbauer polynomials $C_{n}^{\nu}$.

The elementary Cauchy kernel is now given by

$$
\frac{1}{w-z}=\sum_{n=0}^{\infty} A_{n, \nu}(w) z^{-\nu} J_{\nu+n}(z) \text { for }|z|<|w|
$$

This follows from expanding $\frac{z^{\nu}}{w-z}$ in powers of $z$ and writing $z^{\mu}$ for non-negative-integer $\mu$ in terms of Bessels (82 $\$ 5.2)$.

$$
z^{\mu}=\sum_{n=0}^{\infty} \frac{(\mu+2 n) 2^{\mu} \Gamma(\mu+n)}{n !} J_{\mu+2 n}(z) \quad \text { for } \quad \mu \notin \mathbb{Z}_{<0}
$$

Other kernels may also be constructed in closed form (for example, see (123) and (124) below).

These polynomials may be used to construct the dual space for the right sector. To this end we define for all $n \in \mathbb{Z}_{\geq 0}$

$$
\begin{aligned}
\psi_{n, \nu}\left(m e^{i x}\right) & \equiv Z_{n} \frac{e^{-i \nu x}}{m^{\nu}} J_{\nu+n}\left(m e^{i x}\right) \\
\chi_{n, \nu}\left(m e^{i x}\right) & \equiv \frac{m e^{i x}}{Z_{n}} A_{n, \nu}\left(m e^{i x}\right)
\end{aligned}
$$

where $Z_{n}$ is a normalization to be chosen for convenience, later (see (121)). As explicit series

$$
\begin{aligned}
& \psi_{n, \nu}\left(m e^{i x}\right)=\frac{Z_{n}}{2^{\nu+n}} m^{n} e^{i n x} \sum_{k=0}^{\infty} \frac{(-1)^{k}}{k ! \Gamma(1+k+\nu+n)}\left(\frac{m}{2}\right)^{2 k} e^{2 i k x} \\
& \chi_{n, \nu}\left(m e^{i x}\right)=\frac{2^{\nu+n}(\nu+n)}{Z_{n} m^{n}} e^{-i n x} \sum_{k=0}^{\lfloor n / 2\rfloor} \frac{\Gamma(\nu+n-k)}{k !}\left(\frac{m}{2}\right)^{2 k} e^{2 i k x}
\end{aligned}
$$

Both sets of functions are clearly $2 \pi$-periodic. The energy eigenfunctions obey Schrödinger's equation

$$
H \psi_{n, \nu}\left(m e^{i x}\right)=\left(-i \frac{d}{d x}+\nu\right)^{2} \psi_{n, \nu}\left(m e^{i x}\right)+m^{2} e^{2 i x} \psi_{n, \nu}\left(m e^{i x}\right)=(\nu+n)^{2} \psi_{n, \nu}\left(m e^{i x}\right)
$$

while the finite polynomials obey the inhomogeneous differential equation

$$
\begin{aligned}
\left(\widetilde{H}-(\nu+n)^{2}\right) \chi_{n, \nu}\left(m e^{i x}\right) & =\left(-i \frac{d}{d x}-\nu\right)^{2} \chi_{n, \nu}\left(m e^{i x}\right)+\left\{m^{2} e^{2 i x}-(\nu+n)^{2}\right\} \chi_{n, \nu}\left(m e^{i x}\right) \\
& =\frac{2^{\nu}(\nu+n)}{Z_{n}}\left\{\begin{array}{c}
\frac{\Gamma\left(\nu+\frac{1}{2}(n+1)\right)}{\Gamma\left(1+\frac{1}{2}(n-1)\right)} \times 2 m e^{i x} \text { for } n \text { odd } \\
\frac{\Gamma\left(\nu+\frac{1}{2} n\right)}{\Gamma\left(1+\frac{1}{2} n\right)} \times m^{2} e^{2 i x} \text { for } n \text { even }
\end{array}\right.
\end{aligned}
$$


The two sets of functions satisfy the biorthonormality condition

$$
\frac{1}{2 \pi} \int_{0}^{2 \pi} \chi_{k, \nu}\left(m e^{i x}\right) \psi_{n, \nu}\left(m e^{i x}\right) d x=\delta_{k n} \quad \text { for } \quad k, n \in \mathbb{Z}_{\geq 0}
$$

The proof for $k=n$ is obvious from the series (117). The proof for $k \neq n$ proceeds by first acting with $H$ on $\psi_{n, \nu}$ under the integral, then integrating by parts to act with $\widetilde{H}$ on $\chi_{k, \nu}$, and finally comparing the results, i.e. just following the same line of argument as in the case of a single exponential potential, (54) et seq. But note the importance of the $\nu$ sign change for $\widetilde{H}$ compared to $H$.

Thus for any $\nu \notin \mathbb{Z}_{<0}$ we have obtained for the right sector a biorthogonal system that is manifestly in one-to-one correspondence to that of the $\nu=0$ situation: $\left\{\chi_{k, \nu}, \psi_{n, \nu}\right\} \longleftrightarrow\left\{A_{k}, J_{n}\right\}$. We leave the construction of explicit integral transformations between the $\nu \neq 0$ and the $\nu=0$ systems as an exercise. (Hint: see 82 \$11.3)

On the dual space of the right sector, the bilocal norm kernel can once again be obtained in closed form if we choose

$$
Z_{n}^{2}=\frac{\sqrt{4 \pi}}{2^{\nu} \Gamma(\nu+1 / 2)} \frac{(\nu+n) \Gamma(2 \nu+n)}{n !}
$$

Then the hermitian kernel is

$$
J(x, y)=\sum_{n=0}^{\infty} \psi_{n, \nu}\left(m e^{-i x}\right) \psi_{n, \nu}\left(m e^{i y}\right)=\frac{J_{\nu}\left(m e^{-i x}-m e^{i y}\right)}{\left(m e^{-i x}-m e^{i y}\right)^{\nu}}
$$

This follows from the addition theorem $(82 \oint 11.4)$

$$
\frac{J_{\nu}(w-z)}{(w-z)^{\nu}}=\frac{\sqrt{4 \pi}}{2^{\nu} \Gamma(\nu+1 / 2)} \sum_{n=0}^{\infty} \frac{(\nu+n) \Gamma(2 \nu+n)}{n !} w^{-\nu} J_{\nu+n}(w) z^{-\nu} J_{\nu+n}(z)
$$

Note that the ratio $\frac{J_{\nu}(w-z)}{(w-z)^{\nu}}$ is analytic in $w$ and $z$ for all $\nu . \quad J(x, y)$ clearly reduces to the previous case (78) when $\nu=0$.

It is straightforward to act on this norm kernel with the Hamiltonian to obtain a closed form for the Hamiltonian kernel on the dual space, as we did earlier for $\nu=0$. We find

$$
\begin{aligned}
H(x, y) & \equiv\left(\left(-i \partial_{y}+\nu\right)^{2}+m^{2} e^{2 i y}\right) \frac{J_{\nu}\left(m e^{-i x}-m e^{i y}\right)}{\left(m e^{-i x}-m e^{i y}\right)^{\nu}} \\
& =\nu^{2} J(x, y)+(1+2 \nu) m^{2} e^{-i x+i y} \frac{J_{\nu+1}\left(m e^{-i x}-m e^{i y}\right)}{\left(m e^{-i x}-m e^{i y}\right)^{\nu+1}} \\
& =\sum_{n=0}^{\infty}(\nu+n)^{2} \psi_{n, \nu}\left(m e^{-i x}\right) \psi_{n, \nu}\left(m e^{i y}\right)
\end{aligned}
$$

Once again, this is an hermitian kernel, $H(x, y)=H^{*}(y, x)$, and it smoothly reduces to the flux-free $\Phi=0$ case when $\nu=0$, as in (86).

These kernels may be used to compute norms and energy averages, just as in (83) and (88), for arbitrary states in the right sector,

$$
\psi(x)=\sum_{n=0}^{\infty} c_{n} \psi_{n, \nu}\left(m e^{i x}\right)
$$

through the use of the corresponding dual functions

$$
\psi_{\text {dual }}(x)=\sum_{n=0}^{\infty} c_{n}^{*} \chi_{n, \nu}\left(m e^{i x}\right)
$$

Other kernels and corresponding expectation values can be easily constructed, including some like $-i \partial_{y} J(x, y)$ which are non-hermitian. But we will not pursue this here. 


\section{Two exponentials}

This is a complex version of the Morse potential [55]. With a constant vector potential included, the Hamiltonian is

$$
H=(p+\nu)^{2}+\mu_{1} \exp (i x)+\mu_{2} \exp (2 i x)
$$

The analysis of the classical solutions proceeds as before, for fixed energy, and yields trajectories in terms of known functions. We forego further discussion of the classical motion (except in a special case given below) and consider immediately the quantum system.

The corresponding Schrödinger energy eigenvalue equation is

$$
\left[\left(-i \frac{d}{d x}+\nu\right)^{2}+\mu_{1} \exp (i x)+\mu_{2} \exp (2 i x)\right] \psi_{E}(x)=E \psi_{E}(x)
$$

Single-valued solutions, bounded on the real line, exist for any $E \geq 0$ and are given by

$$
\begin{aligned}
\psi_{E}^{ \pm}(x) & =z^{-\nu-\frac{1}{2}} \text { WhittakerM }\left(\frac{1}{2} \frac{\mu_{1}}{\sqrt{-\mu_{2}}}, \pm \sqrt{E}, 2 \sqrt{-\mu_{2}} z\right) \quad \text { where } z \equiv e^{i x} \\
& =e^{(-\nu \pm \sqrt{E}) i x} e^{-\sqrt{-\mu_{2}} e^{i x}} M\left(\frac{1}{2} \pm \sqrt{E}-\frac{1}{2} \frac{\mu_{1}}{\sqrt{-\mu_{2}}}, 1 \pm 2 \sqrt{E}, 2 \sqrt{-\mu_{2}} e^{i x}\right)
\end{aligned}
$$

These eigenfunctions are all manifestly even under PT, for $\nu, \mu_{1}$, and $\sqrt{-\mu_{2}}$ all real. As indicated by the notation, there is a double degeneracy for generic $E$. Here $M(a, b, z) \equiv \operatorname{KummerM}(a, b, z)$ is Kummer's confluent hypergeometric function (1] Chapter 13) also known as ${ }_{1} F_{1}(a ; b ; z)$.

$$
M(a, b, z)={ }_{1} F_{1}(a ; b ; z)=\sum_{n=0}^{\infty} \frac{\Gamma(a+n)}{\Gamma(a)} \frac{\Gamma(b)}{\Gamma(b+n)} \frac{z^{n}}{n !}
$$

So the quantum energy spectrum of this Hamiltonian is the positive real line for any choice of $\mu_{1}$ and $\mu_{2}$. Since it is not always possible to shift $x$ to obtain both real $\mu_{1}$ and real $\mu_{2}$, simultaneously, this model is not translationally equivalent to a PT symmetric theory, in general, and therefore the model provides an interesting counter-example to the naive dictate that real energies require PT symmetry. ${ }^{14}$

The model defines a biorthogonal system for all $E \geq 0$, and for generic $E$ the dual space can be constructed using the methods of the previous example involving only one exponential. In particular, if we restrict our attention to eigenfunctions periodic on $x \in[0,2 \pi]$, we again find exceptional biorthogonal systems. Due to the pre-factor, $e^{(-\nu \pm \sqrt{E}) i x}, 2 \pi$-periodicity requires $-\nu \pm \sqrt{E}=n \in \mathbb{Z}$. So the energies are given in pairs by

$$
E_{ \pm n}=(\nu \pm n)^{2} \quad \text { for } \quad n=0,1, \cdots
$$

This is exactly the same as in the previous model involving only one exponential. For generic $\nu$ the eigenfunctions again define left and right sectors, as in the single exponential case. The dual space can be constructed accordingly.

If we again restrict our attention to the right sector, for simplicity, the dual space may be constructed from polynomials in $1 / z$. For general $\mu_{1}$ and $\mu_{2}$ the relevant polynomials have been investigated by Erdélyi 38. We defer a discussion of their structure for general $\mu_{1}$ and $\mu_{2}$ until the next section of the paper, where we give a unified treatment for all Hamiltonians of the form (11). For special choices of $\mu_{1} \neq 0 \neq \mu_{2}$, however, the biorthogonal structure simplifies dramatically, and many results can again be obtained in closed form. If we set $\nu=0$ and if we make a particular choice for the coefficients of the two exponentials, somewhat surprisingly the eigenfunctions can again be expressed in terms of Bessel functions.

\footnotetext{
${ }^{14}$ However, the model is clearly continuously connected to PT invariant theories through independent variations of both $\mu_{1}$ and $\mu_{2}$. All such variations are isospectral deformations of $H$, i.e. they do not change the total set of energy eigenvalues even though they change the form of $H$ and individual energy eigenfunctions. Relatedly, such variations define continuous deformations of $\mathcal{P} \mathcal{T}$. This statement should be compared to various theorems in the literature on the interplay between general anti-linear symmetries and the reality of the energy spectrum [9] 59 61 14 .
} 
Simplification for a particular two-exponential case Let

$$
H=p^{2}+m e^{i x}-m^{2} e^{2 i x}
$$

Analysis of the classical solutions yields trajectories in terms of elementary functions as in the case of one exponential. For $E>0$, the complex momentum trajectories are now "limaçons" (for examples, see [83]) centered on the Re $p$ axis, rather than the circles of the previous case involving only one exponential. There is also considerably more structure in the $E=0$ classical solutions. Simple special cases occur when $e^{-i x(0)}=m$, for which cases we encounter turning points.

We now consider the quantum system and immediately note the Darboux factorization [30] of the Hamiltonian.

$$
H=-\frac{d^{2}}{d x^{2}}+m e^{i x}-m^{2} e^{2 i x}=\left(-i \frac{d}{d x}-m e^{i x}\right)\left(-i \frac{d}{d x}+m e^{i x}\right)
$$

Schrödinger's eigenvalue equation, the allowed energy eigenvalues, and the periodic eigenfunctions for $x \in$ $[0,2 \pi]$ are now given by

$$
\begin{gathered}
-\frac{d^{2}}{d x^{2}} \psi_{n}(x)+\left(m e^{i x}-m^{2} e^{2 i x}\right) \psi_{n}(x)=n^{2} \psi_{n}(x) \\
\psi_{n}(x)=Z_{n}\left(\frac{m e^{i x}}{2}\right)^{n}\left\{\sum_{k=0}^{\infty} \frac{1}{k ! \Gamma\left(k+\frac{1}{2}+n\right)}\left(\frac{m e^{i x}}{2}\right)^{2 k}-\sum_{k=0}^{\infty} \frac{1}{k ! \Gamma\left(k+\frac{3}{2}+n\right)}\left(\frac{m e^{i x}}{2}\right)^{2 k+1}\right\} \\
=Z_{n} \sqrt{\frac{m e^{i x}}{2}}\left\{I_{n-1 / 2}\left(m e^{i x}\right)-I_{n+1 / 2}\left(m e^{i x}\right)\right\} \\
=\frac{Z_{n}}{\sqrt{2}}\left(-i \frac{d}{d x}-m e^{i x}+1+n\right) \frac{I_{n+1 / 2}\left(m e^{i x}\right)}{\sqrt{m e^{i x}}}
\end{gathered}
$$

for all integer $n$, both positive and negative. $Z_{n}$ is a normalization constant. ${ }^{15}$ The $I$ s are modified Bessel functions, as given by the explicit series (93), or, because in this case they have semi-integer indices, as given by finite sums of powers multiplying hyperbolic functions of $m e^{i x}([1] \S 10.2)$. For $n \in \mathbb{Z}_{n \geq 0}$

$$
I_{n+1 / 2}(z)=\sqrt{\frac{2}{\pi}} z^{n+1 / 2}\left(\frac{1}{z} \frac{d}{d z}\right)^{n} \frac{\sinh z}{z}, \quad I_{-n-1 / 2}(z)=\sqrt{\frac{2}{\pi}} z^{n+1 / 2}\left(\frac{1}{z} \frac{d}{d z}\right)^{n} \frac{\cosh z}{z}
$$

The non-degenerate ground state is simply $\psi_{n=0}(x)=Z_{0} e^{-m e^{i x}} / \sqrt{\pi}$. The result $H \psi_{n=0}(x)=0$ follows immediately from $\left(-i \frac{d}{d x}+m e^{i x}\right) e^{-m e^{i x}}=0$ and the factorized form (133), just as in supersymmetric QM 30.

Duals for all the energy eigenfunctions may be constructed from linear combinations of $\psi_{n \leq 0}$ and $\psi_{n \geq 0}$, similar to (??). On the other hand, if we restrict to the right sector, i.e. the subspace spanned by $\left\{\psi_{n} \mid n \geq 0\right\}$, we may once again make use of only finite polynomials in $e^{-i x}$ to construct the dual space. Explicit calculation gives

$$
\begin{aligned}
\chi_{n}(x) & =\frac{1}{Z_{n}}\left(\frac{2}{m e^{i x}}\right)^{n}\left\{\sum_{k=0}^{\lfloor n / 2\rfloor} \frac{(-1)^{k} \Gamma\left(n-k+\frac{1}{2}\right)}{k !}\left(\frac{m e^{i x}}{2}\right)^{2 k}+\sum_{k=0}^{\lfloor(n-1) / 2\rfloor} \frac{(-1)^{k} \Gamma\left(n-k-\frac{1}{2}\right)}{k !}\left(\frac{m e^{i x}}{2}\right)^{2 k+1}\right\} \\
& =\frac{1}{Z_{n}} \frac{i m e^{i x}}{\sqrt{2}}\left\{\frac{i^{n}}{(n+1 / 2)} A_{n, 1 / 2}\left(i m e^{i x}\right)+\frac{i^{n-1}}{(n-1 / 2)} A_{n-1,1 / 2}\left(i m e^{i x}\right)\right\}
\end{aligned}
$$

These $\left\{\chi_{n} \mid n \geq 0\right\}$ are biorthonormalized duals for $\left\{\psi_{n} \mid n \geq 0\right\}$. By construction, we have

$$
\frac{1}{2 \pi} \int_{0}^{2 \pi} \chi_{k}(x) \psi_{j}(x)=\delta_{k j}
$$

\footnotetext{
${ }^{15}$ The choice $Z_{n}=4^{n} \Gamma\left(\frac{1}{2}+n\right)$ gives a balanced asymptotic behavior for both bilocal norm kernels, $J(x, y)$ and $K(x, y)$.
} 
for all $j, k \geq 0$. The dual functions are now solutions to the inhomogeneous equations

$$
-\frac{d^{2}}{d x^{2}} \chi_{n}(x)+\left(m e^{i x}-m^{2} e^{2 i x}\right) \chi_{n}(x)-n^{2} \chi_{n}(x)=\alpha_{n} m^{2} e^{2 i x}+\beta_{n} m e^{i x}
$$

where the coefficients of the inhomogeneous terms are given by

$$
\begin{array}{cc}
\alpha_{n=2 k}=\frac{(-1)^{k+1} \Gamma\left(k+\frac{1}{2}\right)}{k ! Z_{2 k}}, & \beta_{n=2 k}=-(2 k+1) \alpha_{n=2 k} \\
\alpha_{n=2 k+1}=\frac{(-1)^{k+1} \Gamma\left(k+\frac{1}{2}\right)}{k ! Z_{2 k+1}}, & \beta_{n=2 k+1}=2 k \alpha_{n=2 k+1}
\end{array}
$$

The result $\int_{0}^{2 \pi} \chi_{k}(x) \psi_{j}(x) d x=0$ for $k \neq j$ again follows from integrating by parts and from $\int_{0}^{2 \pi} e^{i x} \psi_{j}(x) d x=$ $0=\int_{0}^{2 \pi} e^{2 i x} \psi_{j}(x) d x$ for all $j \geq 0$, as in (54) et seq.

The construction of the bilocal norm and Hamiltonian kernels proceeds as before. Acting on the dual space of the right sector, we find $\left(w \equiv m e^{-i x}, z \equiv m e^{i y}\right)$

$$
\begin{aligned}
J(x, y) & =\sum_{n=0}^{\infty} \overline{\psi_{n}(x)} \psi_{n}(y) \\
& =\frac{1}{2 \sqrt{w z}}\left[\left(w \frac{d}{d w}-w\right)\left(z \frac{d}{d z}-z\right)+\frac{1}{4}\right] \sum_{n=0}^{\infty}\left|Z_{n}\right|^{2} I_{n+1 / 2}(w) I_{n+1 / 2}(z) \\
& +\frac{1}{2 \sqrt{w z}} \sum_{n=0}^{\infty}\left|Z_{n}\right|^{2} n(n+1) I_{n+1 / 2}(w) I_{n+1 / 2}(z) \\
& +\frac{1}{2 \sqrt{w z}}\left[w \frac{d}{d w}-w+z \frac{d}{d z}-z\right] \sum_{n=0}^{\infty}\left|Z_{n}\right|^{2}\left(n+\frac{1}{2}\right) I_{n+1 / 2}(w) I_{n+1 / 2}(z)
\end{aligned}
$$

The choice $\left|Z_{n}\right|^{2}=(2 n+1) \pi$ facilitates evaluation of the sums, since $(82 \S 11.41 \operatorname{Eqn}(6))$

$\frac{\sinh \sqrt{w^{2}+z^{2}-2 w z s}}{\sqrt{w^{2}+z^{2}-2 w z s}}=\sqrt{\frac{\pi}{2}} \frac{I_{1 / 2}\left(\sqrt{w^{2}+z^{2}-2 w z s}\right)}{\sqrt[4]{w^{2}+z^{2}-2 w z s}}=\frac{\pi}{\sqrt{w z}} \sum_{n=0}^{\infty}(n+1 / 2) I_{n+1 / 2}(w) I_{n+1 / 2}(z)(-1)^{n} P_{n}(s)$

This result, along with $\left.(-1)^{n} P_{n}(s)\right|_{s=-1}=1$ and $\left.2(-1)^{n} \frac{d}{d s} P_{n}(s)\right|_{s=-1}=-n(n+1)$, leads to

$$
\begin{gathered}
\frac{\pi}{\sqrt{w z}} \sum_{n=0}^{\infty}(n+1 / 2) I_{n+1 / 2}(w) I_{n+1 / 2}(z)=\frac{\sinh (w+z)}{w+z} \\
\frac{\pi}{\sqrt{w z}} \sum_{n=0}^{\infty}(n+1 / 2) n(n+1) I_{n+1 / 2}(w) I_{n+1 / 2}(z)=\left(\cosh (z+w)-\frac{\sinh (z+w)}{(z+w)}\right) \frac{2 w z}{(w+z)^{2}}
\end{gathered}
$$

Unfortunately, for the time being, a utilitarian closed form for the last sum in (141) eludes us. We have only found an integral representation for it (see [72, Vol. II, 5.7.17, Eqn(11), p 675).

Given a sufficiently simple result for that third sum, a closed form for the bilocal Hamiltonian $H(x, y)$ would then follow by acting with $H$ on $J(x, y)$, as in the previous cases involving one exponential.

Canonical integral representations for the wave functions, and their duals, can also be exhibited for the two exponential model, especially in the simplified case (132). The easiest of these is probably Poisson's integral (see 11 10.1.13 and 10.1.14 with $z \rightarrow i z$ ), or alternatively just (47) with $\sqrt{E}$ replaced by $n+1 / 2$ and $m \rightarrow i m$ in the argument of the Bessel function. Corresponding integral representations for the duals follow from biorthonormality, for given choices of the kernels (e.g. for Poisson's integral, an appropriate kernel is given by [1 10.2.36). This allows construction of a Table as in the case of the one exponential model. We leave the details of this construction to the well-motivated, interested reader. 


\section{$7 \quad$ Biorthogonal systems for $H=p^{2}+\sum_{k>0} \mu_{k} \exp (i k x)$}

We indicate methods to analyze the general case, reserving some of the details to be presented elsewhere. For $x \in(-\infty,+\infty)$ without any periodicity constraints, the spectrum of this Hamiltonian is known 42 ] to be the real, positive half-line, for any choice of $\mu \mathrm{s} \mathrm{such}$ that $\sum_{k>0}\left|\mu_{k}\right|<\infty$, and not just for those $\mu \mathrm{s}$ which are real or $x$-translationally equivalent to real values. So PT symmetry is not required for real energy eigenvalues in this model. Here, we will restrict our attention to $2 \pi$-periodic functions and their duals, to obtain a discrete subset of real energy eigenvalues, namely just $\left\{E_{n}=n^{2} \mid n=0,1, \cdots\right\}$.

A simple procedure to construct this biorthogonal system is to begin with the dual functions. ${ }^{16}$ We assume these to be polynomials in powers of $z^{-1} \equiv e^{-i x}$, so we write

$$
\chi_{n}(z)=\frac{1}{z^{n}} \sum_{j=0}^{n} c_{n, j} z^{j}, \quad H=\left(z \frac{d}{d z}\right)^{2}+\sum_{k>0} \mu_{k} z^{k}
$$

These polynomials, for three or more $\mu \mathrm{s}$, would seem to be a natural generalization of those constructed by Neumann, Gegenbauer, and Erdélyi. Acting with $H-n^{2}$ gives

$$
\left(z \frac{d}{d z}\right)^{2} \chi_{n}(z)-n^{2} \chi_{n}(z)+\sum_{k>0} \mu_{k} z^{k} \chi_{n}(z)=\frac{1}{z^{n}} \sum_{j=1}^{n}\left((j-n)^{2}-n^{2}\right) c_{n, j} z^{j}+\sum_{k>0} \mu_{k} \frac{z^{k}}{z^{n}} \sum_{j=0}^{n} c_{n, j} z^{j}
$$

We now impose the condition that the RHS involve only positive powers of $z$ so as to obtain an inhomogeneity that will be orthogonal to the span of $\left\{z^{n} \mid n \geq 0\right\}$ under contour integration $\oint \frac{d z}{z} \cdots$, as in previous examples. This leads to $n$ equations that fix the coefficients $c_{n, k}$ for $k=1, \cdots, n$ in terms of $c_{n, 0}$, the latter being an overall choice of normalization. Explicitly, the powers to be eliminated, and the resulting "triangular" set of $n$ equations, are

$$
\begin{array}{cl}
z^{-n+1}: & c_{n, 1}\left((1-n)^{2}-n^{2}\right)+\mu_{1} c_{n, 0}=0 \\
z^{-n+2}: & c_{n, 2}\left((2-n)^{2}-n^{2}\right)+\mu_{2} c_{n, 0}+\mu_{1} c_{n, 1}=0 \\
\vdots & \vdots \\
z^{-n+n}: & c_{n, n}\left((n-n)^{2}-n^{2}\right)+\mu_{n} c_{n, 0}+\mu_{n-1} c_{n, 1}+\cdots+\mu_{1} c_{n, n-1}=0
\end{array}
$$

These equations can always be solved, sequentially, for $c_{n, k}$ in terms of $c_{n, 0}$ and the $\mu \mathrm{s}$, as expressed by the recursion

$$
c_{n, k}=\frac{1}{(2 n-k) k} \sum_{j=0}^{k-1} \mu_{k-j} c_{n, j}, \quad k=1, \cdots, n .
$$

So for a given $n$ the $c_{n, k}$ will depend on $\mu_{j \leq n}$ but not on $\mu_{j>n}$. Indeed, the $c_{n, k}$ are finite polynomials in the $\mu \mathrm{s}$, and therefore non-singular for all $\mu \mathrm{s}$.

Thus, up to their individual normalizations, the dual functions are completely determined along with the inhomogeneous equations that they obey, without (yet!) having to find the eigenfunctions of $H$. The resulting equation giving the action of $H$ on the dual functions is of the form

$$
\left(H-n^{2}\right) \chi_{n}(z)=\sum_{k>0} \gamma_{n, k} z^{k}
$$

where the coefficients $\gamma_{n, k}$ may also be expressed explicitly as finite polynomials in the $\mu \mathrm{s}$,

$$
\gamma_{n, k}=\sum_{j=0}^{n} \mu_{k+n-j} c_{n, j}
$$

\footnotetext{
${ }^{16}$ So these systems are an exception to the statement: "A direct and frontal attack on the problem of determining [the dual polynomials] is not usually fruitful." - Morse and Feshbach [56 p 931.
} 
This result follows from (145) and (147). Note that $k$ is not necessarily limited by $n$ in $\gamma_{n, k}$. The $\gamma_{n, k}$ will depend on all the $\mu$ s, in general, with $k$ taking on all values up to and including the highest power of $z$ appearing in $H$.

With $\left\{\chi_{n}(z)\right\}$ in hand, we may then proceed to construct $\left\{\psi_{n}(z)\right\}$ as analytic functions around the origin by demanding biorthonormality with $\left\{\chi_{n}(z)\right\}$. We take the $\psi_{n}$ functions to be infinite series of positive powers

$$
\psi_{n}(z)=z^{n} \sum_{j=0}^{\infty} a_{n, j} z^{j}
$$

If we assume the requisite convergence of these series, their forms automatically give $\oint \frac{d z}{z} \chi_{k}(z) \psi_{n}(z)=$ 0 for $n>k$. Otherwise, conditions are obtained on the coefficients $a_{n, j}$ for $j+n \leq k$ by requiring $\frac{1}{2 \pi i} \oint \frac{d z}{z} \chi_{k}(z) \psi_{n}(z)=\delta_{k n}$ for every $k \geq n$. These conditions amount to another triangular set of equations which can always be solved, sequentially, for the $a_{n, j}, j \leq k-n$, for any choice of $\mu \mathrm{s}$. Namely

$$
a_{n, 0}=\frac{1}{c_{n, 0}}, \quad \sum_{j=0}^{k-n} c_{k, k-n-j} a_{n, j}=0 \quad \text { for } \quad k>n
$$

By considering all $k>n$ in succession, we obtain all $a_{n, j}$. The series for $\psi_{n}$ is a development in the minors that invert these triangular equations. For convenience we choose $c_{n, 0}=1$ for all $n$, then

$$
\begin{aligned}
& \chi_{n}(z)=\frac{1}{z^{n}}\left(1+c_{n, 1} z+c_{n, 2} z^{2}+\cdots+c_{n, n} z^{n}\right) \\
& \psi_{n}(z)=z^{n}-c_{n+1,1} z^{n+1}+\left|\begin{array}{cc}
c_{n+1,1} & 1 \\
c_{n+2,2} & c_{n+2,1}
\end{array}\right| z^{n+2}-\left|\begin{array}{ccc}
c_{n+1,1} & 1 & 0 \\
c_{n+2,2} & c_{n+2,1} & 1 \\
c_{n+3,3} & c_{n+3,2} & c_{n+3,1}
\end{array}\right| z^{n+3}+\cdots
\end{aligned}
$$

The coefficients of $z^{n+k}$ in $\psi_{n}(z)$ are again finite polynomials in the $\mu$ s. While convergence of this series is certainly not obvious for arbitrary $\mu \mathrm{s}$, as written, it is clear that convergence can be determined on a case-by-case basis from the explicit form of the coefficients. Moreover, when the number of $\mu \mathrm{s}$ is finite, no matter what their values are, it is not too difficult to show the $\psi_{n}(z)$ are entire functions of $z$.

Thus, if we assume the requisite convergence of the $\psi_{n}$ series, all the $\left\{\psi_{n}(z) \mid\right.$ for $\left.n \geq 0\right\}$ are determined, and they are complete on the span of $\left\{z^{n} \mid n \geq 0\right\}$. All positive powers of $z$ can be expressed as series of $\left\{\psi_{n}(z)\right\}$, similar in form to (20), just as all negative powers of $z$ can be expressed as finite sums of $\left\{\chi_{n}(z)\right\}$, similar in form to (21).

Remarkably, the $\left\{\psi_{n}(z)\right\}$ just obtained turn out to be non-degenerate energy eigenfunctions, and are in fact all of the $2 \pi$-periodic eigenfunctions of $H$, with

$$
H \psi_{n}(z)=n^{2} \psi_{n}(z)
$$

The RHS of (154) can be deduced in a novel way from (148), the biorthogonality of $\left\{\chi_{j}(z), \psi_{k}(z)\right\}$, and the completeness of $\left\{\psi_{n}(z)\right\}$ for analytic functions about the origin, as follows. Given that $\psi_{n}(z)$ is such an analytic function, as in (150), completeness allows us to write $H \psi_{n}(z)=\sum_{k=n}^{\infty} b_{n, k} \psi_{k}(z)$. From this and biorthonormality, we have $b_{n, k}=\frac{1}{2 \pi i} \oint \frac{d z}{z} \chi_{k}(z) H \psi_{n}(z)$. But then, upon integrating by parts and using (148) as well as orthogonality relations, we also have $\frac{1}{2 \pi i} \oint \frac{d z}{z} \chi_{k}(z) H \psi_{n}(z)=n^{2} \delta_{k, n}$. So $b_{n, k}=n^{2} \delta_{k, n}$, and (154) is obtained. ${ }^{17}$

Conversely, given (154) and (148), we may prove the orthogonality relations $\oint \frac{d z}{z} \chi_{k}(z) \psi_{n}(z)=0$ for $k \neq n$ just by inserting $H$ and following the same line of argument as in the case of a single exponential potential, (54) et seq. Some explicit closed forms for the various functions, some kernels constructed from sums of their bilinears, and related convergence issues, as well as many other details for this general case, will be discussed elsewhere.

\footnotetext{
${ }^{17}$ Note that $z=0$ is a regular singular point of [154), for any number and any values of the $\mu$ s such that $\sum_{k>0} \mu_{k} z^{k}$ converges near the origin. In fact, 153 is exactly the conventional series obtained by expanding about the regular singular point at $z=0$, albeit the series was obtained here in an unusual way from the properties of the dual space polynomials. Convergence criteria for 153) in terms of the $\mu$ s are obtained in the conventional series approach in 7342 , without any discussion of the dual polynomials.
} 


\section{A brief look at QFT extensions}

For the complex Liouville quantum field theory in $1+1$ spacetime, we choose to define the Hamiltonian density by normal-ordering at mass $m$ in the standard way [23].

$$
\mathcal{H}=N_{m}\left(\frac{1}{2} \pi_{\psi}^{2}+\frac{1}{2}\left(\partial_{\sigma} \psi\right)^{2}+\mu e^{2 i \beta \psi}\right)
$$

Normal-ordering at any other mass should not affect our conclusions. The resulting (pseudo)scalar field theory is conformally covariant [29], 16], at least for a range of $\beta$. For this field theory, the only issue to be raised here is the stability of the ground state. Hollowood [48 has previously discussed separate but related issues in his study of solitons for complex Toda theories using conventional Hilbert space methods and perturbation theory.

We may attempt to place an upper bound on the ground state $\langle\mathcal{H}\rangle$ using the conventional Hilbert space norm through an adaptation [24] of the variational argument of $\S I I I$ in Coleman 23]. Take as a trial state the zero-mode-shifted Fock vacuum for arbitrary mass $M$, defined by the effect of annihilation operators for that mass:

$$
a(0, M)|0, \xi, M\rangle=\frac{1}{2} \xi|0, \xi, M\rangle, \quad a(k, M)|0, \xi, M\rangle=0 \quad \text { if } \quad k \neq 0
$$

For $|0, \xi, M\rangle$ the zero-mode configuration is coherent, while the $a(k, m \neq M)$ non-zero mode configuration is superfluidic (i.e. built formally from powers of correlated pairs of creation operators $\left(a^{\dagger}(k, m) a^{\dagger}(-k, m)\right)^{n}$ acting on $|0,0, m\rangle)$. Note that

$$
\left\langle 0, \xi, M\left|N_{m}\left(e^{2 i \beta \psi}\right)\right| 0, \xi, M\right\rangle=e^{2 i \beta \xi}\left\langle 0, \xi, M\left|N_{m}(\cos (2 \beta(\psi-\xi)))\right| 0, \xi, M\right\rangle
$$

since $\left\langle 0, \xi, M\left|N_{m}(\sin (2 \beta(\psi-\xi)))\right| 0, \xi, M\right\rangle=0$. For this trial state, we find

$$
\langle 0, \xi, M|\mathcal{H}| 0, \xi, M\rangle=\mu e^{2 i \beta \xi}\left(\frac{M^{2}}{m^{2}}\right)^{\beta^{2} / 2 \pi}+\frac{1}{8 \pi}\left(M^{2}-m^{2}\right)
$$

Therefore, by choosing $\xi$ such that $\mu e^{2 i \beta \xi}$ is real and negative, and then letting $M / m \rightarrow \infty$, there is no lower bound on $\langle 0, \xi, M|\mathcal{H}| 0, \xi, M\rangle$ if $\beta^{2}>2 \pi$. Thus in the usual Fock space framework, there is no ground state if $\beta^{2}$ exceeds $2 \pi$. This may indicate a phase-transition for the field theory.

For the field theoretic extension corresponding to the model (10), with $\nu=0$, the RHS of (158) becomes just a sum over $k=2 \beta$ with various $\mu_{k}$. Thus the variational argument shows an instability whenever the sum over $k$ includes $k>5$.

The problem is now to determine how this variational argument is effected by using the bilocal norm instead of the usual Hilbert space norm. The operator methods in [18 and/or the functional methods in 27] should suffice for this purpose. There have been studies of the variational principle for similar "quasihermitian" theories [75] and the techniques introduced in those studies should be applicable to expectations of (155). This issue will be fully discussed elsewhere.

Here we only remark that the situation is reminiscent of $-\phi^{4}$ field theory, which also has no ground state based on conventional variational arguments using the standard Hilbert space norm and trial states with real expectations $\langle\phi\rangle$, but which appears to be an acceptable model when viewed as PT symmetric $\phi^{2}(i \phi)^{2}$ theory [10]. This is unquestionably related to the use of the CPT norm, but more directly, it is due to the definition of the zero-mode problem on a contour in the LHP, and not the real axis. This obviates most physical intuition about the ground state of that theory, in our opinion. Even so, there are obviously major differences between PT symmetric $-\phi^{4}$ field theory and imaginary Liouville theory as defined by (155), in that the apparent instability in $-\phi^{4}$ field theory is evident at the classical level for any coupling strength, whereas the instability indicated by (158) for $\beta^{2}>2 \pi$ is a purely quantum effect.

\section{Conclusions}

We made a thorough analysis of some complex one dimensional periodic potentials, of Liouville type, within the framework of biorthogonal systems. We provided an extensive guide to the considerable literature for 
these models. We conclude that these systems are consistent at both classical and quantum mechanical levels, with remarkable and deep connections between the two levels, just as in the case of real Liouville models. In fact, the connections are more intimate in the complex case, inasmuch as the classical structure is richer. We studied in some detail the correspondence and impact of the classical dynamics on that of the quantum problem.

More specifically we have shown that the complex Liouville model is equivalent to a projected free particle system, and we may choose that free particle projection to be chiral. With that choice, the complex Hamiltonian may be viewed as a completely local description for the free chiral particle, the only such local Hamiltonian description known to us.

We have used the remarkable properties of Neumann polynomials as the biorthogonal partners of Bessel functions on a circle in the complex plane. With those properties in hand, we obtained closed forms for the inner product and various other hermitian kernels. We enlarged this study to include a wide class of complex periodic one dimensional Hamiltonians, and we obtained similar closed form results in several additional cases.

In our analysis, the theory of PT symmetric models was often a guide but did not play a decisive role insofar as the states were self-orthogonal in the periodic sectors that we emphasized and some of the models that we considered were not PT symmetric. Moreover, many of the concepts that we used are already contained in the previously existing framework of biorthogonal systems. As stressed in the title of the paper, the use of biorthonormal systems of vectors for the construction of consistent quantum mechanical systems was the real conceptual lift, applicable even when the states are self-orthogonal. The most important feature shared with the PT symmetric approach was simply that of a complex Hamiltonian with real eigenvalues.

It would be interesting to investigate biorthogonal systems for self-orthogonal subsectors of other solvable complex potential models, e.g. those in [51, for which the duals would seem to be the associated functions described in [80]. Another interesting and possibly important challenge is to combine the biorthogonal approach with the study of symmetries of the Hamiltonian. Beyond the usual commutators between any local symmetry generators, there must also be commutation relations among the various bilocal kernels. Thus the full set of symmetries of the corresponding quantum problem may not be local and may not be obvious. Along these lines, it is perhaps a useful working hypothesis that any consistent local complex Hamiltonian with real eigenvalues is related by a similarity transformation to that of a hermitian, albeit nonlocal, Hamiltonian. However, this conceptual device may not always lead to practical calculational tools as this similarity transformation is usually not known, a priori, and may be difficult to implement when it is known.

A clear and readable discussion on the use of similarity transformations to expose the hermitian underpinnings of certain apparently non-hermitian (i.e. "quasi-hermitian") theories is given in [75] (also see 63, 64, 66, 67]). The general theory is nicely illustrated in a recent paper on PT-symmetric $-z^{4}$ quantum mechanics 49, where after the change of variable $z=-2 i \sqrt{1+i x}, x \in \mathbb{R}$, the relevant similarity transformation is very simple and easily implemented.

While substantial progress has already been made on several PT symmetric field theories [5], 57, it is important to apply the methods of biorthogonal systems to more general quantum field theories and many-body problems, particularly those of the complex Liouville and Toda type. It remains a challenge to consider situations involving self-orthogonal states in these models, to compute correlation functions using those states, and to investigate their possible relevance for string theories.

The study of the present paper may lead to a deeper appreciation of the behavior of quantum Liouville field theory for complex coupling constants, as sketched in Section 8. This would further understanding of subcritical string theory, especially the correlators for those models which exhibit interesting structure in the complex coupling plane (cf. 69] and references therein, especially [36, 87]). In fact, Liouville theory with imaginary coupling (or its close cousin under complex continuation - so-called "timelike" Liouville theory) has already been analyzed using conformal field theory methods $[78$, 76. We believe the Hamiltonian analysis initiated above will help shed additional light on this subject.

Moreover, the field theory extensions of the models described in Section 7 are relevant to applications of conformal field theory and phase-transitions. In particular, complex Liouville field theory may be useful to understand freezing transitions 21, 22, More recently, there is very interesting work [2] involving complex Liouville field theory in non-Hermitian Luttinger liquids and flux line pinning in planar superconductors. As for other possible physical applications of the techniques in this paper, it is perhaps interesting to conjecture 
that coupled Bose-Einstein systems with dissipative effects might be governed by relatively simple nonhermitian effective Hamiltonians. This could be, in several respects, a completely quantum analogue of the classical driven/damped oscillator systems discussed in [47].

Acknowledgements We thank C Bender for introducing us to PT symmetric theories and raising our awareness of non-hermitian Hamiltonian methods. We also thank P G O Freund, A Veitia, and D Schuster for useful discussions. One of us (TC) thanks the Aspen Center for Physics and the Institute for Advanced Study for providing stimulating environments in which this work was completed. Finally, we thank the anonymous referee for making helpful suggestions and for bringing some relevant papers to our attention. This material is based upon work supported by the National Science Foundation under Grant No. 0303550.

\section{References}

[1] M Abramowitz and I A Stegun, editors, Handbook of mathematical functions, with formulas, graphs, and mathematical tables, Appl. Math. Ser. No. 55, National Bureau of Standards, US GPO, 1964.

[2] I Affleck, W Hofstetter, D R Nelson, and U Schollwock, J. Stat. Mech. 0410:P003, 2004 cond-mat/0408478.

[3] S Banach, Theory of Linear Operations, North-Holland, 1987 (reprint of the 1932 Warsaw edition).

[4] N K Bari, "Biorthogonal systems and bases in Hilbert space" Moskov. Gos. Univ. Učen. Zap. 148, Matematika 4 (1951), 69-107. (Russian) MR 14, 289.

[5] C Bender, "Introduction to PT-Symmetric Quantum Theory" quant-ph/0501052.

This review provides a guide to the PT literature, with emphasis on the contributions of the author and his collaborators.

[6] C M Bender, S Boettcher, and P N Meisinger, J. Math. Phys. 40 (1999) 2201-2229 quant-ph/9809072.

[7] C M Bender, S Boettcher, H F Jones, and V M Savage, J. Phys. A: Math. Gen. 32 (1999) 6771-6781 quant-ph/9906057.

[8] C Bender, G V Dunne, and P N Meisinger, Phys. Lett. A252 ( 1999) 272-276 cond-mat/9810369.

[9] C M Bender, P N Meisinger, and Q Wang, J. Phys. A: Math. Gen. 36 (2003) 1029 quant-ph/0211123.

[10] C M Bender, P Meisinger, and H Yang, Phys. Rev. D63 (2001) 45001.

[11] C Bender and Q Wang, J. Phys. A: Math. Gen. 34 (2001) 3325-3328.

[12] G D Birkhoff, Trans. Amer. Math. Soc. 9 (1908) 373-395.

[13] B. Birnir, Comm. Pure Appl. Math. 39 (1986) 1-49.

[14] A Blasi, G Scolarici, and L Solombrino, J. Phys. A: Math. Gen. 37 (2004) 4335-4351 quant-ph/0310106.

[15] E Braaten, T Curtright, G Ghandour, and C Thorn, Phys. Rev. Lett. 51 (1983) 19-22; Annals Phys. 153 (1984) 147-201.

[16] E Braaten, T Curtright, G Ghandour, and C Thorn, Phys. Lett. B125 (1983) 301-304. This paper discusses conformal covariance for some $1+1$ field theories without assuming hermitian interactions.

[17] E Braaten, T Curtright, and C Thorn, Phys. Lett. B118 (1982) 115-120.

[18] E Braaten, T Curtright, and C Thorn, Annals Phys. 147 (1983) 365-416.

[19] C Brezinski, Biorthogonality and its applications to numerical analysis, M Dekker, 1992. This book is available in an on-line edition, and provides a modern guide to the earlier literature. 
[20] F Cannata, G Junker, and J Trost, Phys. Lett. A246 (1998) 219-226 quant-ph/9805085.

[21] D Carpentier and P Le Doussal, Phys. Rev. E63 (2001) 026110 cond-mat/0003821.

[22] H Castillo and P Le Doussal, Phys. Rev. Lett. 86 (2001) 4859 cond-mat/0006737.

[23] S Coleman, Phys Rev D11 (1975) 2088-2097.

[24] T Curtright, unpublished (1975). "It is curious to recall some of the mental habits of those departed days." - H G Wells (1898)

[25] T Curtright, "Quantum Bäcklund Transformations and Conformal Algebras" pp 279-289 in the proceedings of the 18th Int. Conf. on Differential Geometric Methods in Theoretical Physics: Physics and Geometry, Tahoe City, CA, Jul 2-8, 1989.

[26] T Curtright, "Schrödinger's Cataplex," pp 121-132, proceedings of the 1999 Coral Gables conference quant-ph/0011101.

[27] T Curtright and G Ghandour, "Using Functional Methods to Compute Quantum Effects in the Liouville Model" published in Coral Gables QFT 1991:333-345 (QC174.45:N21:1991) hep-th/9503080.

[28] T Curtright, L Mezincescu, and A Veitia, to appear.

[29] T Curtright and C Thorn, Phys. Rev. Lett. 48 (1982) 1309-1313 ; Erratum-ibid. 48 (1982)1768.

[30] G Darboux, C. R. Hebd. Seances Acad. Sci. 94 (1882) 1456; M M Crum, Q. J. Math. 6 (1955) 121; P Deift, Duke Math. J. 45 (1978) 267; E Witten, Nucl. Phys. B188 (1981) 513; F Cooper, A Khare, and U Sukhatme, Phys. Rep. 251 (1995) 267 hep-th/9405029; C Bender, F Cooper, P Meisinger, and V M Savage, Phys. Lett. A259 (1999) 224-231 quant-ph/9907008.

[31] P Deift, unpublished (ca. 1985).

[32] E D'Hoker and R Jackiw, Phys. Rev. D26 (1982) 3517-3542.

[33] J Dieudonné, Michigan J. Math. 2 (1954) 7-20.

[34] P A M Dirac, Rev. Mod. Phys. 17 (1945) 195-199.

[35] P Dorey, C Dunning, and R Tateo, J. Phys. A: Math. Gen. 34 (2001) 5679-5704 hep-th/0103051.

[36] H Dorn and H J Otto, Nucl. Phys. B429 (1994) 375 hep-th/9403141.

[37] N Dunford, Bull. Amer. Math. Soc. 64 (1958) 217-274; see also N Dunford and J T Schwartz, Linear Operators, Part III, Spectral Operators, John Wiley \& Sons, 1971.

[38] A Erdélyi, Monatshefte Math. Phys. 47 (1939) 87-103. It is almost always useful to consult original papers, but especially in this instance, since the paper is mis-cited as well as mis-quoted by the Bateman Manuscript Project, despite the latter being edited by the paper's author.

[39] D B Fairlie and J Nuyts, J. Phys. A: Math. Gen. 38 (2005) 3611-3624 hep-th/0412148.

[40] F H M Faisal and J V Moloney, J. Phys. B 14 (1981) 3603-3620.

[41] H Feshbach, C E Porter, and V Weisskopf, Phys. Rev. 96 (1954) 448-464.

[42] M G Gasymov, Funct. Anal. Appl. 14 (1980) 11-15.

[43] G I Ghandour, Phys. Rev. D35 (1987) 1289-1295.

[44] I T Gohberg and M G Krein, Introduction to the Theory of Linear Nonselfadjoint Operators in Hilbert Space, Nauka, 1965. 
[45] E Goursat, Cours d'analyse mathématique, Gauthier-Villars, 1923-1924. Vol. 3, 4. éd., rev. et augm., 1927. Available in English translation, Dover Publications [1959-64].

[46] M C Gutzwiller, J. Math. Phys. 12 (1971) 343-358.

[47] W D Heiss, J. Phys. A: Math. Gen. 37 (2004) 2455-2464 quant-ph/0304152.

[48] T Hollowood, Nucl. Phys. B384 (1992) 523-540 hep-th/9110010.

[49] H F Jones and J Mateo "An Equivalent Hermitian Hamiltonian for the non-Hermitian $-x^{4}$ Potential" quant-ph/0601188

[50] T Kato, Perturbation Theory for Linear Operators, Springer, 1995.

[51] A Khare and U Sukhatme, Phys. Lett. A324 (2004) 406-414 quant-ph/0402106; ; "PT-Invariant Periodic Potentials with a Finite Number of Band Gaps" math-ph/0505027.

[52] J R Klauder and B Skagerstam, Coherent states: applications in physics and mathematical physics, World Scientific, 1985.

[53] D C McGarvey, J. Math. Anal. Appl. 4 (1962) 366-410; 11 (1965) 564-569; 12 (1963) 187-234.

[54] G A Mezincescu, J. Phys. A: Math. Gen. 33 (2000) 4911-4916; J. Phys. A: Math. Gen. 34 (2001) 3329-3332. quant-ph/0002056

[55] P M Morse, Phys. Rev. 34 (1929) 57-64.

[56] P M Morse and H Feshbach, Methods of Theoretical Physics, McGraw-Hill, 1953.

[57] A Mostafazadeh, "Pseudo-Hermiticity, PT-symmetry, and the Metric Operator" quant-ph/0508214. This review provides another guide to the literature, with emphasis on the contributions of the author and his collaborator.

[58] A Mostafazadeh, J. Math. Phys. 43 (2002) 205-214 math-ph/0107001

[59] A Mostafazadeh, J. Math. Phys. 43 (2002) 2814-2816 math-ph/0110016

[60] A Mostafazadeh, J. Math. Phys. 43 (2002) 3944-3951 math-ph/0203005

[61] A Mostafazadeh, J. Math. Phys. 44 (2003) 974-989 math-ph/0209018

[62] A Mostafazadeh, J. Math. Phys. 45 (2004) 932-946 math-ph/0302050

[63] A Mostafazadeh, J. Math. Phys. 46 (2005) 102108 quant-ph/0506094.

[64] A Mostafazadeh, J. Phys. A: Math. Gen. 36 (2003) 7081-7092 quant-ph/0304080.

[65] A Mostafazadeh, J. Phys. A: Math. Gen. 38 (2005) 3213-3234 quant-ph/0410012.

[66] A Mostafazadeh, J. Phys. A: Math. Gen. 38 (2005) 6557-6569 quant-ph/0411171.

[67] A Mostafazadeh and A Batal, J. Phys. A: Math. Gen. 37 (2004) 11645-11680 quant-ph/0408132.

[68] N I Muskhelishvili, Singular Integral Equations, Appendix 3, P. Noordhoff, 1953.

[69] Y Nakayama, Int. J. Mod. Phys. A1 (2004) 2771-2930, and references cited therein hep-th/0402009.

[70] C G Neumann, Theorie der Bessel'schen Functionen, B G Teubner, Leipzig, 1867.

[71] L A Pastur and V.A. Tkachenko, Funct. Anal. Appl. 22 (1988) 156-158.

[72] A P Prudnikov, Y A Brychkov, and O I Marichev, Integrals and series, Gordon and Breach Science Publishers, 1986. 
[73] F S Rofe-Beketov, Soviet Math. Dokl. 4 (1963) 1563-1566.

[74] B F Samsonov, J. Phys. A: Math. Gen. 38 (2005) L571-L579 quant-ph/0507085; B F Samsonov and P Roy, J. Phys. A: Math. Gen. 38 (2005) L249-L255 quant-ph/0503040.

[75] F G Scholtz, H B Geyer, and F J W Hahne, Ann. Phys. 213 (1992) 74-101.

[76] V Schomerus, JHEP 0311 (2003) 043 hep-th/0306026; S Fredenhagen and V Schomerus, JHEP 0505 (2005) 025 hep-th/0409256.

[77] K C Shin, J. Math. Phys. 42 (2001) 2513-2530; Math. Nachr. 261-262 (2003) 171-175; J. Math. Anal. Appl. 299 (2004) 19-3; J. Phys. A: Math. Gen. 37 (2004) 8287-8292 math-ph/0007006 math-ph/0308032 math.SP/0312254 math-ph/0404015.

[78] A Strominger and T Takayanagi, Adv. Theor. Math. Phys. 7 (2003) 369-379 hep-th/0303221; M Gutperle and A Strominger, Phys. Rev. D67 (2003) 126002 hep-th/0301038.

[79] V A Tkachenko, Sov. Math. Dokl. 5 (1964) 413-415.

[80] O A Veliev, Sov. Math. Dokl. 250 (1980) 1292-1296; O A Veliev and M Toppamuk Duman, J. Math. Anal. Appl. 265 (2002) 76-90.

[81] N Vilenkin, Special functions and the theory of group representations, American Mathematical Society, 1968.

[82] G N Watson, Theory of Bessel Functions, Cambridge University Press, 2nd Edition, 1944.

[83] E W Weisstein, "Limacon." http://mathworld.wolfram.com/Limacon.html

[84] J Wong, J. Math. Phys. 8 (1967) 2039-2042.

[85] S Youssef, "Physics with exotic probability theory" hep-th/0110253.

For other papers along these lines, see http://physics.bu.edu/ youssef/quantum/quantum_refs.html

[86] C Zachos, D Fairlie, and T Curtright, Quantum Mechanics in Phase Space, World Scientific, 2005.

[87] A B Zamolodchikov and A B Zamolodchikov, Nucl. Phys. B477 (1996) 577 hep-th/9506136.

[88] M Znojil, Phys. At. Nucl. 65 (2002) 1149-1151; "New types of solvability in PT symmetric quantum theory" math-ph/0501058. 